Check for updates

Cite this: RSC Adv., 2020, 10, 27874

Received 2nd July 2020

Accepted 20th July 2020

DOI: $10.1039 / \mathrm{dOra05768a}$

rsc.li/rsc-advances

\section{Preparation and facile addition reactions of iminium salts derived from amino ketene silyl acetal and amino silyl enol ether $\dagger$}

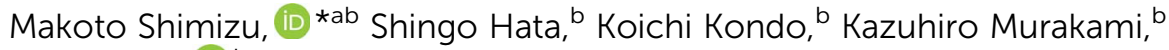 \\ Isao Mizota (iD ${ }^{\mathrm{b}}$ and Yusong Zhu ${ }^{\mathrm{a}}$
}

While iminium salts generated by the oxidation of amino ketene silyl acetals show intriguing reactivities to give useful $\gamma$-oxo- $\alpha$-amino esters via reactions with silyl enol ethers in good yields, new iminium salts are also prepared by the oxidation of amino silyl enol ethers. They undergo facile addition reaction with various nucleophiles to give $\alpha$-amino ketone derivatives in good yields.

\section{Introduction}

Iminium salts are usually very reactive species and used widely in organic synthesis. Several methods are known to generate these useful species. Among them the synthesis of natural products, such as alkaloids, was reported using cyclization mediated by iminium salts as a crucial step. ${ }^{1}$ Representative examples using iminium species involve the addition of organometallic reagents for the synthesis of $\beta$-amino acids, ${ }^{2 a, b}$ $\beta$-amino ketones, 1,3-amino alcohols, ${ }^{2 c}$ and so on. ${ }^{2 d-j}$ The synthesis of $\alpha$-monosubstituted $\alpha$-amino acids was reported by the nucleophilic addition of boronates to the iminium ions, which were prepared by the condensation of primary or secondary amines with ethyl glyoxylate in situ. ${ }^{3}$ Although the use of iminium salts is a promising procedure, it is not trivial to generate these species in a chemo- and regioselective manner under mild reaction conditions.

We have been interested in the generation of iminium salts and have already disclosed several intriguing features. ${ }^{4}$ In these studies, we focused on the generation of iminium species by oxidizing a readily accessible and stable enol derivative with oxidants and found that the alkoxycarbonyl iminium salts from amino ketene silyl acetals were reasonably stable ${ }^{5}$ and highly reactive to give addition products with metal cyanides, Grignard reagents, allyl metals, ${ }^{4 b}$ ketene silyl acetals, ${ }^{4 g}$ and indoles ${ }^{4 c}$ (eqn (1), Scheme 1). However, we have not fully studied reactions with silyl enol ethers, since an initial examination using the silyl enol ether derived from cyclopentanone met with a disappointing result where only a trace amount of the addition product was obtained.

${ }^{a}$ School of Energy Science and Engineering, Nanjing Tech University, Nanjing 211816, Jiangsu Province, China

${ }^{b}$ Department of Chemistry for Materials, Graduate School of Engineering, Mie University, Tsu, Mie 514-8507, Japan. E-mail: mshimizu@chem.mie-u.ac.jp

$\dagger$ Electronic supplementary information (ESI) available. See DOI: 10.1039/d0ra05768a
We now reexamined the reaction with other silyl enol ethers and found that an acceptable range of product yields was obtained under carefully controlled conditions. We did not study generation and reaction of the iminium salts derived from amino silyl enol ethers, either, although these iminium salts are highly attractive in terms of reactivity and chemoselectivity on an addition reaction with nucleophiles. This paper describes addition reactions of the alkoxycarbonyl iminium salts with silyl enol ethers to produce $\gamma$ oxo- $\alpha$-amino esters (eqn (2)). Reactions of the iminium salts derived from amino silyl enol ethers with various nucleophiles are also presented to give addition products (eqn (3)).

\section{Results and discussion}

The starting amino ketene silyl acetal $\mathbf{1}$ was readily prepared according to the reported procedures in good overall yield. ${ }^{4 b, f}$

\section{Previous work}

(1)
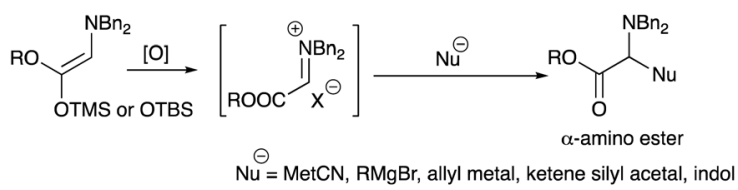

2. Present work

(2)

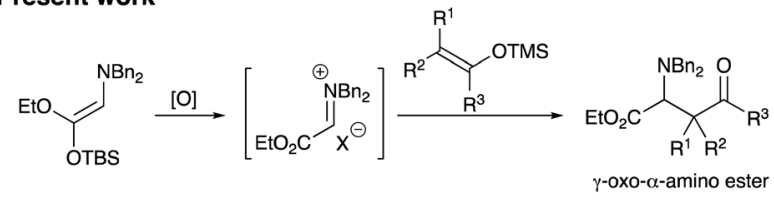

(3)
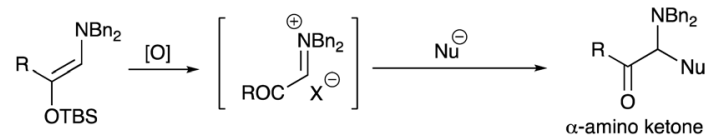

$\mathrm{Nu}=\mathrm{RMgBr}$, ketene silyl acetal, indol 
Table 1 Reaction of the iminium salt with cyclic ketene silyl acetals

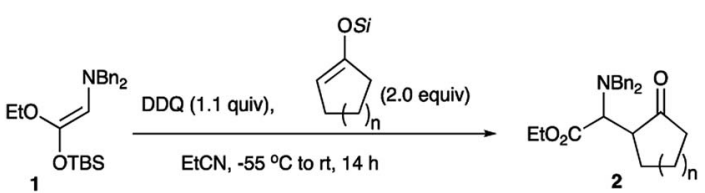

\begin{tabular}{lllll}
\hline Entry & $n$ & $\mathrm{Si}^{a}$ & Yield $^{b}(\%)$ & syn/anti \\
\hline 1 & 1 & TMS & 2a: 9 & $100 / 0$ \\
2 & 2 & TMS & 2b: 73 & $66 / 34$ \\
3 & 2 & TBS & 2b: 59 & $45 / 55$ \\
4 & 2 & TIPS & 2b: 61 & $45 / 55$ \\
5 & 3 & TMS & 2c: 71 & $57 / 43$ \\
6 & 4 & TMS & 2d: 68 & $60 / 40$
\end{tabular}

${ }^{a}$ Abbrebiations, TMS: trimethylsilyl, TBS: tert-butyldimethylsilyl, TIPS: triisopropylsilyl. ${ }^{b}$ Isolated yield.

First, silyl enol ethers derived from cyclic ketones were subjected to the addition reaction, and Table 1 summarizes the results.

Although an initial examination was carried out with the TMS enol ether derived from cyclopentanone, only a small amount of the desired addition product was obtained (entry 1). Switching the ring size from 5 to 6,7 , and 8 resulted in the formation of the desired products in 73,71 , and $68 \%$ yields, respectively (entries 2, 5, and 6). We next examined the effect of the silyl substituent on the diastereoselectivity. ${ }^{6}$ However, the

Table 2 Reaction of the iminium salt with acyclic ketene silyl acetals

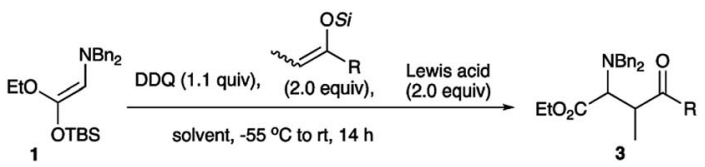

\begin{tabular}{|c|c|c|c|c|c|c|c|}
\hline Entry & $\mathrm{R}$ & $\mathrm{Si}$ & $Z / E$ & Solv. & Lewis acid & Yield $(\%)^{a}$ & syn/anti \\
\hline 1 & Cy & TMS & $85 / 15$ & EtCN & - & 3a: 32 & $52 / 48$ \\
\hline 2 & Сy & TMS & $85 / 15$ & DMF & - & 3a: 67 & $77 / 23$ \\
\hline 3 & $\mathrm{Cy}$ & TMS & $85 / 15$ & DME & $\mathrm{BF}_{3} \cdot \mathrm{Et}_{2} \mathrm{O}$ & 3a: 22 & $76 / 24$ \\
\hline 4 & Сy & TBS & $85 / 15$ & DMF & - & 3a: 62 & $78 / 22$ \\
\hline 5 & Cy & TBS & $85 / 15$ & DME & $\mathrm{BF}_{3} \cdot \mathrm{Et}_{2} \mathrm{O}$ & 3a: 25 & $65 / 35$ \\
\hline 6 & $\mathrm{Ph}$ & TMS & $90 / 10$ & DME & $\mathrm{BF}_{3} \cdot \mathrm{Et}_{2} \mathrm{O}$ & 3b: 57 & $57 / 43$ \\
\hline 7 & $\mathrm{Ph}$ & TMS & $90 / 10$ & DME & $\mathrm{Et}_{2} \mathrm{AlCl}$ & $3 \mathbf{b}: 30$ & $66 / 34$ \\
\hline 8 & $\mathrm{Ph}$ & TMS & $90 / 10$ & DMF & - & 3b: 78 & $51 / 49$ \\
\hline 9 & $\mathrm{Ph}$ & TBS & $90 / 10$ & DME & - & $3 \mathbf{b}: 8$ & $60 / 40$ \\
\hline 10 & $\mathrm{Ph}$ & TBS & $90 / 10$ & DME & $\mathrm{BF}_{3} \cdot \mathrm{Et}_{2} \mathrm{O}$ & 3b: 38 & $58 / 42$ \\
\hline 11 & $\mathrm{Ph}$ & TBS & $90 / 10$ & DME & $\mathrm{Et}_{2} \mathrm{AlCl}$ & 3b: 31 & $72 / 28$ \\
\hline 12 & $\mathrm{Ph}$ & TBS & $90 / 10$ & DMF & - & 3b: 64 & $56 / 44$ \\
\hline 13 & $\mathrm{Ph}$ & TIPS & $85 / 15$ & DME & - & $3 b: 13$ & $92 / 8$ \\
\hline 14 & $\mathrm{Ph}$ & TIPS & $85 / 15$ & DME & $\mathrm{BF}_{3} \cdot \mathrm{Et}_{2} \mathrm{O}$ & $3 b: 60$ & $86 / 14$ \\
\hline 15 & $\mathrm{Ph}$ & TIPS & $85 / 15$ & DME & $\mathrm{Et}_{2} \mathrm{AlCl}$ & $3 b: 30$ & $88 / 12$ \\
\hline 16 & $\mathrm{Ph}$ & TIPS & $85 / 15$ & DMF & - & 3b: 79 & $60 / 40$ \\
\hline 17 & $\mathrm{Ph}$ & $\mathrm{DMS}^{b}$ & $88 / 12$ & DME & - & 3b: 57 & $73 / 27$ \\
\hline 18 & $\mathrm{Ph}$ & DMS & $88 / 12$ & DME & $\mathrm{BF}_{3} \cdot \mathrm{Et}_{2} \mathrm{O}$ & $3 b: 40$ & $51 / 49$ \\
\hline 19 & $\mathrm{Ph}$ & DMS & $88 / 12$ & DMF & - & $3 b: 62$ & $59 / 41$ \\
\hline
\end{tabular}

${ }^{a}$ Isolated yield. ${ }^{b}$ Abbrebiation, DMS: dimethylsilyl.
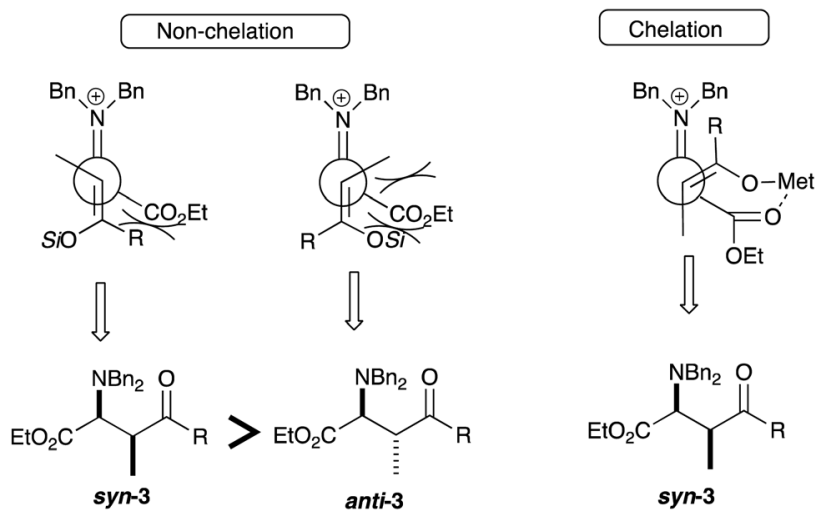

Scheme 2 Possible transition state models.

use of bulky substituents such as TBS and TIPS did not improve the diastereoselectivity (entries 3 and 4). Reactions of acyclic silyl enol ethers were next examined. Table 2 summarizes the results.

In terms of the product yields using the silyl enol ether derived from 1-cyclohexylpropan-1-one, the reaction in EtCN did not give a satisfactory result (entry 1), whereas the use of DMF in the absence of a Lewis acid gave better results (entries 2 and 4). The effects of a Lewis acid are not prominent on the diastereoselctivity, giving the addition products with moderate selectivities (entries 2 to 5). ${ }^{7}$ Regarding the silyl enol ether derived from propiophenone, both TMS and TIPS derivatives in DMF in the absence of a Lewis acid gave high yields of the product (entries 8 and 16). In general, the TIPS derivative recorded good syn-selectivities (entries 13 to 15). Among them the reaction in the presence of a Lewis acid $\left(\mathrm{BF}_{3} \cdot \mathrm{Et}_{2} \mathrm{O}\right.$ or $\mathrm{Et}_{2}$ $\mathrm{AlCl}$ ) gave the syn-adduct with good selectivities (entries 14 and 15), whereas the presence of a Lewis acid destroyed selectivities in certain cases (entries 6, 10, and 18). For the explanation of the syn-selectivity, Scheme 2 shows possible transition state models.

In the absence of a Lewis acid or in the presence of $\mathrm{BF}_{3} \cdot \mathrm{Et}_{2} \mathrm{O}$ (monodentate Lewis acid), the non-chelation model would explain the preferred formation of the syn-isomer 3 due to the less steric congestion. In the cases where the reaction was carried out in the presence of $\mathrm{Et}_{2} \mathrm{AlCl}$ (bidentate Lewis acid), the chelation model would also support the formation of the synisomer 3. On the basis of the above transition state models, the

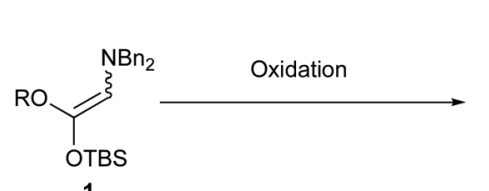

1

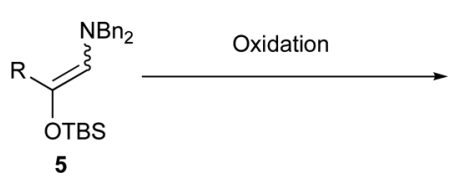

Scheme 3 Generation of a new iminium salt.
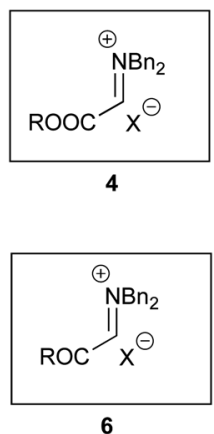


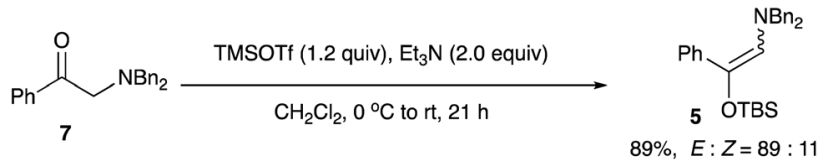

Scheme 4 Preparation of the amino silyl enol ether 5.

formation of the syn-isomer 3 would be preferred regardless of the presence of Lewis acids.

During investigations into the reactivity of iminium salts $\mathbf{4}$ derived from amino ketene silyl acetals, new iminium salts from amino silyl enol ethers intrigued us (Scheme 3). However, no reliable reports are available for the generation of the iminium salts of type 6 . We therefore investigated the generation of this type of iminium salt using a method similar to those from amino ketene silyl acetals.

The amino silyl enol ether $\mathbf{5}$ was readily prepared as follows in good yield (Scheme 4).

First, the formation of the iminium salt followed by addition reactions using the amino silyl enol ether $\mathbf{5}$ was examined to find the best reaction conditions involving the oxidation reagent and a Lewis acid. Table 3 summarizes the results. Iodosylbenzene did not effect the oxidation, while the use of $\mathrm{BPO},{ }^{4 a} \mathrm{NCS}$, and DBDMH gave the desired product in low yields (entries 1 to 4 ). $\mathrm{DDQ}^{4 b, 8}$ which was used for the oxidation of the amino ketene silyl acetal 1 effected the reaction to give the addition product $8 \mathrm{a}$ in $24 \%$ yield (entry 5 ). Among the oxidation reagents examined here the use of NBS recorded the best result (entry 6). ${ }^{4 f}$ We next examined the use of a Lewis acid for the present transformation. $\mathrm{Et}_{2} \mathrm{AlCl}$ was not effective, while an increased amount of the addition product was obtained in the
Table 4 Reaction with various ketene silyl(thio) acetas

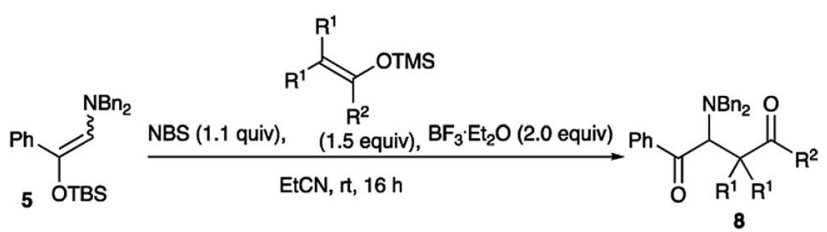

\begin{tabular}{lllll}
\hline Entry & $\mathrm{R}^{1}$ & $\mathrm{R}^{2}$ & Product & Yield $^{a}(\%)$ \\
\hline 1 & $\mathrm{Me}$ & OMe & $\mathbf{8 a}$ & 80 \\
2 & $\mathrm{Me}$ & OEt & $\mathbf{8 b}$ & 82 \\
3 & $\mathrm{Me}$ & OiPr & $\mathbf{8 c}$ & 73 \\
4 & $\mathrm{Me}$ & OtBu & $\mathbf{8 d}$ & 57 \\
5 & $\mathrm{MeO}$ & OMe & $\mathbf{8 e}$ & 73 \\
6 & $\mathrm{EtO}$ & OEt & $\mathbf{8 f}$ & 70 \\
7 & $\mathrm{H}$ & $\mathrm{StBu}$ & $\mathbf{8 g}$ & 63 \\
${ }^{a}$ Isolated yields. & & &
\end{tabular}

presence of $\mathrm{TiCl}_{4}$ (entries 7 and 8). A better result was obtained when the reaction was carried out with NBS in the presence of $\mathrm{BF}_{3} \cdot \mathrm{Et}_{2} \mathrm{O}$, and the addition product was obtained in $74 \%$ yield (entry 9). The amounts of ketene silyl acetal and $\mathrm{BF}_{3} \cdot \mathrm{Et}_{2} \mathrm{O}$ were further examined. The use of the reduced amounts of the ketene silyl acetal (1.5 equiv.) and $\mathrm{BF}_{3} \cdot \mathrm{Et}_{2} \mathrm{O}$ (1.2 equiv.) gave a slightly better result (entry 10). Regarding the reaction temperature, the reaction at a higher temperature gave a reduced amount of the desired product (entry 13). The best result was obtained when the reaction was carried out with the ketene silyl acetal (1.5 equiv.) and $\mathrm{BF}_{3} \cdot \mathrm{Et}_{2} \mathrm{O}$ (2.0 equiv.) at $\mathrm{rt}$, and the addition product 8a was obtained in $80 \%$ yield (entry 15). Under the optimized

Table 3 Examination of the reaction conditions

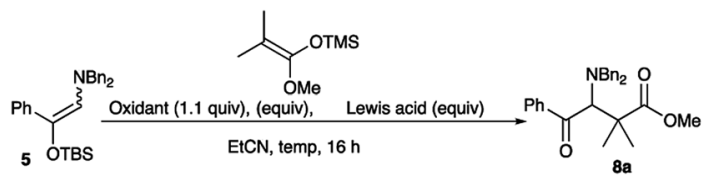

\begin{tabular}{|c|c|c|c|c|c|}
\hline Entry & Oxidant & $\mathrm{Nu}$ (equiv.) & Lewis acid (equiv.) & Temp & Yield $^{a}(\%)$ \\
\hline 1 & PhIO & 2.0 & - & $\mathrm{rt}$ & 0 \\
\hline 2 & BPO & 2.0 & - & $\mathrm{rt}$ & 7 \\
\hline 4 & DBDMH & 2.0 & - & $\mathrm{rt}$ & 10 \\
\hline 5 & DDQ & 2.0 & - & $\mathrm{rt}$ & 24 \\
\hline 6 & NBS & 2.0 & - & $\mathrm{rt}$ & 27 \\
\hline 9 & NBS & 2.0 & $\mathrm{BF}_{3} \cdot \mathrm{Et}_{2} \mathrm{O}(2.0)$ & $\mathrm{rt}$ & 74 \\
\hline 10 & NBS & 1.5 & $\mathrm{BF}_{3} \cdot \mathrm{Et}_{2} \mathrm{O}(1.2)$ & $\mathrm{rt}$ & 76 \\
\hline 11 & NBS & 1.5 & $\mathrm{BF}_{3} \cdot \mathrm{Et}_{2} \mathrm{O}(1.5)$ & $\mathrm{rt}$ & 69 \\
\hline 12 & NBS & 1.2 & $\mathrm{BF}_{3} \cdot \mathrm{Et}_{2} \mathrm{O}(2.0)$ & $\mathrm{rt}$ & 53 \\
\hline 13 & NBS & 1.5 & $\mathrm{BF}_{3} \cdot \mathrm{Et}_{2} \mathrm{O}(2.0)$ & $50^{\circ} \mathrm{C}$ & 40 \\
\hline 14 & NBS & 1.5 & $\mathrm{BF}_{3} \cdot \mathrm{Et}_{2} \mathrm{O}(2.0)$ & $0^{\circ} \mathrm{C}$ to $\mathrm{rt}$ & 77 \\
\hline
\end{tabular}

${ }^{a}$ Isolated yield. 
conditions, various ketene silyl acetals were subjected to this addition reaction, and Table 4 summarizes the results.

As shown in Table 4, tetra-substituted ketene silyl acetals underwent addition reaction readily to give the adducts in good yields. Regarding the ester alkoxy part, methyl and ethyl esters were obtained in good yields (entries 1 and 2), whereas a bulky ${ }^{t}$ butyl derivative recorded a decreased yield of the product (entry 4). Di-substituted thioester also participated in the present addition to give the adduct in moderate yield (entry 7). We next examined the use of indole derivatives as nucleophiles, since indole skeletons are often found in many biologically active compounds such as tryptophan, indomethacin, and dragmacidin derivatives, and several methodologies have been reported to functionalize indoles. ${ }^{9}$ Table 5 summarizes the results.

$N$-TIPS indole was subjected to the present reaction conditions to give a moderate yield of the addition product 9a (entry 1). The best result was obtained when the reaction was conducted at $0{ }^{\circ} \mathrm{C}$ to $\mathrm{rt}$ for $5 \mathrm{~h}$ (entry 2). An electron-withdrawing group, 5-nitro substituent decreased the product yield considerably (entry 5), whereas 5-MeO, 5- $\mathrm{Br}$, and 6- $\mathrm{Br}$ groups did not affect the addition reaction to give the adducts in good yields (entries 6 to 8). However, $N$-H free and $N$-Ts derivatives were not suitable for the present reaction, presumably due to the decreased electron-density at the 3-position of the indole skeleton (entries 9 and 10). We next examined the use of Grignard reagents as nucleophiles.

Treatment of the amino silyl enol ether 5 with NBS followed by ethylmagnesium bromide in the presence of $\mathrm{BF}_{3} \cdot \mathrm{Et}_{2} \mathrm{O}$ actually gave the ethylated product 10b. Table 6 summarizes the optimization of reaction conditions.

As can be seen form Table 6, the best conditions were found when the amino silyl enol ether 5 was treated with NBS (1.1 equiv.) and ethylmagnesium bromide (2.0 equiv.) in the presence of $\mathrm{BF}_{3} \cdot \mathrm{Et}_{2} \mathrm{O}$ (2.0 equiv.) in EtCN at $\mathrm{rt}$ for $30 \mathrm{~min}$, and the ethylation product $\mathbf{1 0 b}$ was obtained in $74 \%$ yield (entry 4 ).

Table 5 Reaction with various indoles

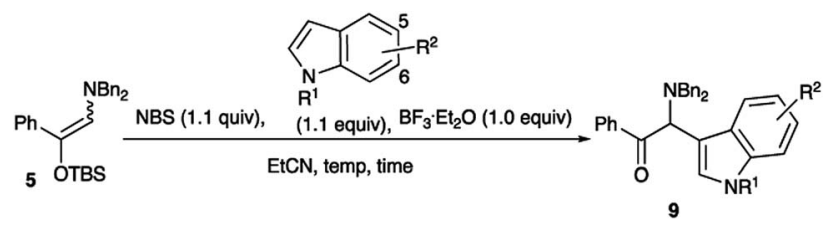

\begin{tabular}{llllll}
\hline Entry & $\mathrm{R}^{1}$ & $\mathrm{R}^{2}$ & Temp $\left({ }^{\circ} \mathrm{C}\right)$ & Time $(\mathrm{h})$ & Yield $^{a}(\%)$ \\
\hline 1 & 9a: TIPS & $\mathrm{H}$ & $\mathrm{rt}$ & 5 & 43 \\
2 & 9a: TIPS & $\mathrm{H}$ & 0 to $\mathrm{rt}$ & 5 & 77 \\
3 & 9a: TIPS & $\mathrm{H}$ & 0 & 3 & 51 \\
4 & 9a: TIPS & $\mathrm{H}$ & 0 & 5 & 70 \\
5 & 9b: TIPS & $5-\mathrm{NO}_{2}$ & 0 & 5 & 13 \\
6 & 9c: TIPS & $5-\mathrm{MeO}$ & 0 & 5 & 67 \\
7 & 9d: TIPS & $5-\mathrm{Br}$ & 0 & 5 & 75 \\
8 & 9e: TIPS & $6-\mathrm{Br}$ & 0 & 5 & 73 \\
9 & 9f: $\mathrm{H}$ & $\mathrm{H}$ & 0 & 5 & 0 \\
10 & 9f: Ts & $\mathrm{H}$ & 0 & 5 & 0
\end{tabular}

${ }^{a}$ Isolated yields.
Table 6 Optimization of the ethylation conditions

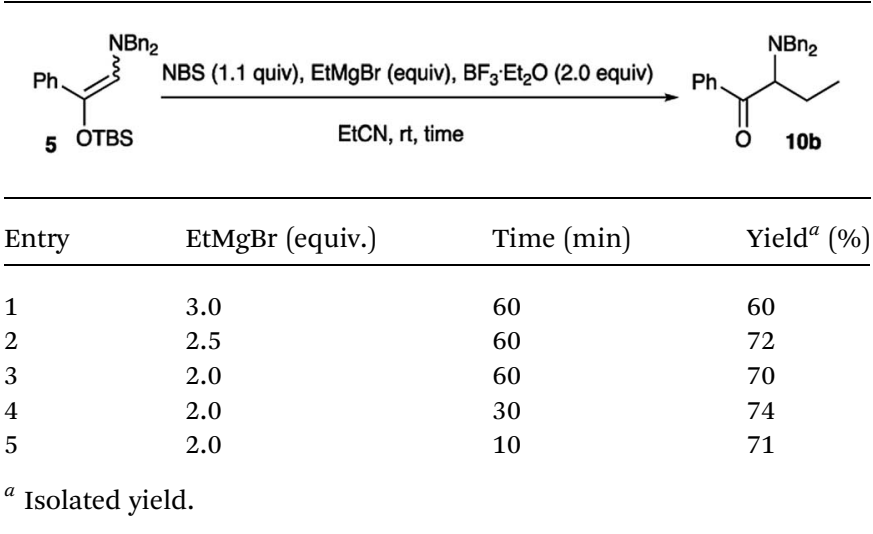

Under the optimized conditions, a variety of Grignard reagents were subjected to the alkylation, and Table 7 summarizes the results.

Methyl, ethyl, npropyl, iso-propyl, cyclohexyl, and 2-thiehyl Grignard reagents recorded good product yields (entries 1-4, 6, and 10), whereas cylopropyl, benzyl, and 4-tolyl derivatives gave the addition products in moderate yields (entries 5, 6, and 9). However, phenyl and ethylnylmagnesium bromides did not give the desired products but gave complex mixtures (entries 8 and 11). Compared with the results obtained from the reactions of the iminium salts generated by the oxidation of amino ketene silyl acetals with Grignard reagents, ${ }^{4 b}$ this $\alpha$-amino ketonederived iminium salt appears to be only a little bit less reactive than ester-derived ones. Thus, the present iminium salt derived from $\alpha$-amino ketone shows good reactivity as an electrophile to give a variety of addition products. The following Scheme 5 shows possible reaction pathways.

Regarding the formation of the iminium salt $\mathbf{4}$ derived from $\alpha$-amino ester, DDQ oxidizes the amino ketene silyl acetal 1 to

Table 7 Alkylation of the iminium salt

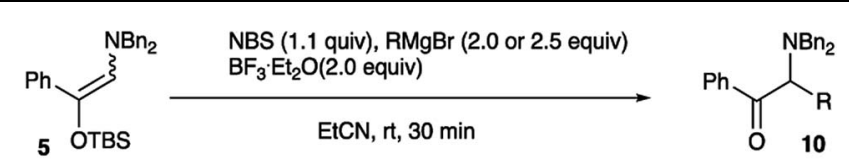

\begin{tabular}{lllll}
\hline Entry & $\mathrm{R}$ & Product & Yield $^{a}(\%)$ & Yield $^{b}(\%)$ \\
\hline 1 & $\mathrm{Me}$ & $\mathbf{1 0 a}$ & 46 & 64 \\
2 & $\mathrm{Et}$ & $\mathbf{1 0 b}$ & 74 & - \\
3 & $n \mathrm{Pr}$ & $\mathbf{1 0 c}$ & 71 & 62 \\
4 & $\mathrm{iPr}$ & $\mathbf{1 0 d}$ & 63 & 63 \\
5 & $\mathrm{cPr}$ & $\mathbf{1 0 e}$ & 27 & 35 \\
6 & $\mathrm{Cy}$ & $\mathbf{1 0 g}$ & 58 & 60 \\
7 & $\mathrm{Bn}$ & $\mathbf{1 0 h}$ & 0 & 43 \\
8 & $\mathrm{Ph}$ & $\mathbf{1 0 i}$ & 46 & 0 \\
9 & $4-\mathrm{MeC}_{6} \mathrm{H}_{4}$ & $\mathbf{1 0 j}$ & 64 & 38 \\
10 & $2-\mathrm{Thienyl}$ & $\mathbf{1 0 k}$ & 0 & 45 \\
11 & Ethynyl & & & 0
\end{tabular}

${ }^{a} \mathrm{RMgBr}$ (2.0 equiv.) was used. ${ }^{b} \mathrm{RMgBr}$ (2.5 equiv.) was used. ${ }^{c} \mathrm{BnMgCl}$ was used. 
(1)<smiles>CCOC(C)=CC(=O)OC</smiles>
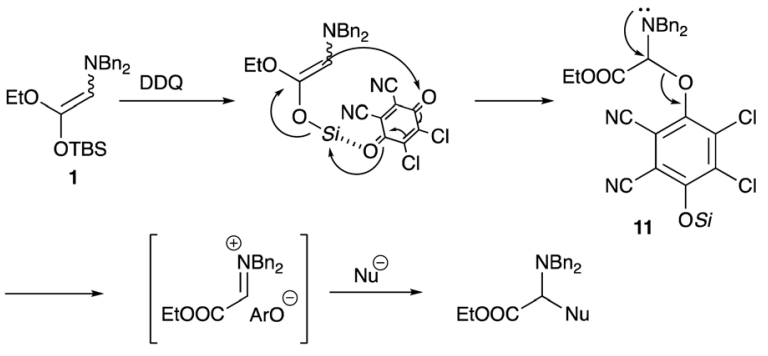

4

(2)
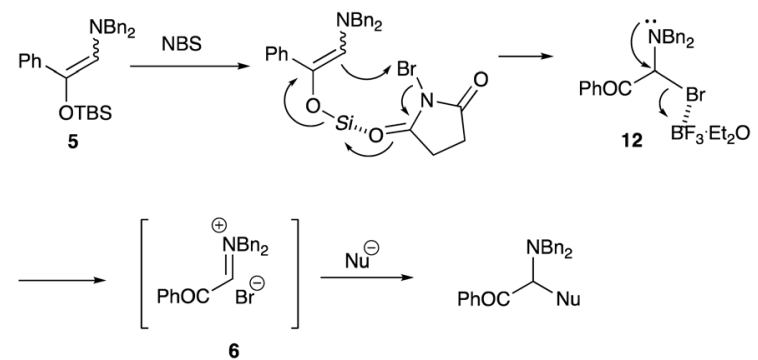

Scheme 5 Proposed reaction pathways.

form the $\mathrm{N}, \mathrm{O}$-acetal 11, which collapses to form the iminium salt 4. This iminium salt 4 is responsible for the formation of the addition products with silyl enol ethers. In the case of $\alpha$ amino ketone, NBS reacts with the amino silyl enol ether 5 to form the bromide 12. An activation with $\mathrm{BF}_{3} \cdot \mathrm{Et}_{2} \mathrm{O}$ promotes the elimination of a $\mathrm{Br}$ ion to form the iminium salt $\mathbf{6}$, which reacts with a variety of nucleophiles.

\section{Conclusions}

In conclusion, the iminium salt prepared from amino ketene silyl acetal showed good reactivity and reacted with silyl enol ethers to give $\gamma$-oxo- $\alpha$-amino esters in good yields. Regarding the diastereoseletivity of the reactions with the silyl enol ethers derived from ethyl ketones, a good syn-selectivitiy was observed. In particular, in the case with the TIPS enol ether derived from propiophenone, the presence of Lewis acids improved the synselectivity. The formation of the iminium salt derived from 2aminoacetophenone was readily carried out via the oxidation of its TBS enol ether with NBS. The presence of $\mathrm{BF}_{3} \cdot \mathrm{Et}_{2} \mathrm{O}$ facilitated the reaction to give addition products in good yields. These results indicate that the iminium salts possessing an electron-withdrawing groups next to the iminium carbon are readily prepared via the oxidation of their trialkylsilyl enolates with certain oxidants. These iminium species are reasonably reactive and act as good acceptors of nucleophiles to give addition products with ketene silyl acetals, indoles, and Grignard reagents in good yields.

\section{Experimental}

\section{General aspects}

Infrared spectra were determined on a JASCO FT/IR-460 plus spectrometer. ${ }^{1} \mathrm{H}$ NMR and ${ }^{13} \mathrm{C}$ NMR spectra were recorded with a JEOL ECX-400P, or a JEOL A-500 spectrometer using tetramethylsilane as an internal standard. Mass spectra were recorded on a JEOL MS-700D spectrometer. Propionitrile (EtCN) was distilled from phosphorus pentaoxide and then from calcium hydride, and stored over molecular sieves 4 A. Dimethyl formamide (DMF) was distilled from calcium hydride and stored over molecular sieves 4 A. Dimethoxyethane (DME) was distilled from calcium hydride and then cupper(I) chloride, and stored over sodium. 1-Ethoxy-2-dibenzylamino-1- ${ }^{t}$ butyldimethylsiloxyethylene $\mathbf{1}^{3 b}$ was synthesized by the reported procedure. Purification of products was performed by column chromatography on silica gel (Kanto Silica Gel 60N) and/or preparative TLC on silica gel (Merck Kiesel Gel GF254 or Wako Gel B-5F).

\section{Synthesis of ethyl 2-(dibenzylamino)-2-(2-oxocyclohexyl) acetate $2 b$ (general procedure for the addition reaction with cyclic silyl enol ethers)}

In a $30 \mathrm{~mL}$ two-necked round-bottomed flask equipped with a magnetic stirring bar, a rubber septum, and an argon balloon was introduced a solution of 2,3-dichloro-5,6-dicyano-1,4benzoquinone (DDQ) $(37.4 \mathrm{mg}, 0.165 \mathrm{mmol})$ in EtCN $(1.0 \mathrm{~mL})$. The solution was cooled to $-55{ }^{\circ} \mathrm{C}$, and to it were added successively solutions of 1-ethoxy-2-dibenzylamino-1- ${ }^{t}$ butyldimethylsiloxyethylene 1 (59.6 mg, $0.150 \mathrm{mmol})$ in EtCN $(1 \mathrm{~mL})$ and 1-cyclohexenyloxytrimethylsilane $(25.0 \mathrm{mg}, 0.150 \mathrm{mmol})$ in EtCN $(1 \mathrm{~mL})$. The reaction mixture was allowed to warm to room temperature with stirring for $14 \mathrm{~h}$. The reaction was quenched with $10 \%$ aq. $\mathrm{Na}_{2} \mathrm{CO}_{3}$, and the whole mixture was extracted with ethyl acetate $(10 \mathrm{~mL} \times 3)$. The combined extracts were washed with brine $(15 \mathrm{~mL})$, dried $\left(\mathrm{Na}_{2} \mathrm{SO}_{4}\right)$, and concentrated in vacuo. The crude product was purified by preparative TLC on silica gel $\left(\mathrm{CH}_{2} \mathrm{Cl}_{2}:\right.$ nhexane $\left.=6: 1\right)$ to give the title compound $(s y n-2 \mathbf{b}$ $(27.2 \mathrm{mg}, 48 \%)$ and anti-2b $(14.0 \mathrm{mg}, 25 \%))$.

Syn-2b. Yield 48\% (27.2 mg); colorless oil; $R_{\mathrm{f}}=0.26$ ( $n$ hexane : ethyl acetate $=5: 1) ;{ }^{1} \mathrm{H}$ NMR $\left(400 \mathrm{MHz}, \mathrm{CDCl}_{3}\right) \delta 1.02-$ $1.61(\mathrm{~m}, 6 \mathrm{H}$ including triplet at $1.40 \mathrm{ppm}, J=7.3 \mathrm{~Hz}, 3 \mathrm{H}), 1.86-$ $2.05(\mathrm{~m}, 2 \mathrm{H}), 2.26-2.35(\mathrm{~m}, 2 \mathrm{H}), 2.59-2.63(\mathrm{~m}, 1 \mathrm{H}), 2.96-2.98$ $(\mathrm{m}, 1 \mathrm{H}), 3.37$ (d, $J=13.7 \mathrm{~Hz}, 2 \mathrm{H}), 3.39$ (d, $J=10.7 \mathrm{~Hz}, 1 \mathrm{H}), 3.86$ $(\mathrm{d}, J=13.7 \mathrm{~Hz}, 2 \mathrm{H}), 4.29(\mathrm{dq}, J=7.3,10.9 \mathrm{~Hz}, 1 \mathrm{H}), 4.30(\mathrm{dq}, J=$ 7.3, $10.9 \mathrm{~Hz}, 1 \mathrm{H}), 7.22-7.37(\mathrm{~m}, 10 \mathrm{H}) ;{ }^{13} \mathrm{C} \mathrm{NMR}(100 \mathrm{MHz}$, $\left.\mathrm{CDCl}_{3}\right) \delta 14.8,25.2,28.1,31.1,42.5$ 50.0, 54.8, 60.3, 60.5, 127.2, 128.4, 129.1, 139.2, 170.9, 212.2; IR (neat) 1712, 1637, 1453, 1176, 1150, 1030, 799, 700, 621, 496, 470, 438, $420 \mathrm{~cm}^{-1}$; HRMS (EI) calcd for $\mathrm{C}_{24} \mathrm{H}_{29} \mathrm{NO}_{3}(\mathrm{M})^{+} 379.2147$, found 379.2164.

Anti-2b. Yield 25\% (14.0 mg); colorless oil; $R_{\mathrm{f}}=0.54$ ( $n$ hexane : ethyl acetate $=5: 1) ;{ }^{1} \mathrm{H}$ NMR $\left(500 \mathrm{MHz} \mathrm{CDCl}_{3}\right) \delta 1.35-$ $1.81(\mathrm{~m}, 10 \mathrm{H}$ including triplet at $1.56 \mathrm{ppm}, J=7.3 \mathrm{~Hz}), 2.02-2.05$ (m, 1H), 3.04-3.08 (m, 1H), $3.32(\mathrm{~d}, J=13.1 \mathrm{~Hz}, 2 \mathrm{H}), 3.88$ (d, $J=$ $11.0 \mathrm{~Hz}, 1 \mathrm{H}), 3.96(\mathrm{~d}, J=13.1 \mathrm{~Hz}, 2 \mathrm{H}), 4.27(\mathrm{dq}, J=7.3,10.7 \mathrm{~Hz}$, $1 \mathrm{H}), 4.35$ (dq, $J=7.3,10.7 \mathrm{~Hz}, 1 \mathrm{H}), 7.22-7.33(\mathrm{~m}, 10 \mathrm{H}) ;{ }^{13} \mathrm{C}$ NMR $\left(126 \mathrm{MHz}, \mathrm{CDCl}_{3}\right) \delta 14.8,20.7,28.0,38.0,49.5,53.6,54.5,60.6$, $61.2,127.3,128.5,129.0,139.2,170.5,219.0$; IR (neat) 2860, 1710, 1638, 1494, 1451, 1373, 1335, 1307, 1233, 1175, 1135, 1027, 969, 794, 744, 699, $501 \mathrm{~cm}^{-1}$; HRMS (EI) calcd for $\mathrm{C}_{24} \mathrm{H}_{29} \mathrm{NO}_{3}(\mathrm{M})^{+}$379.2147, found 379.2148. 


\section{Ethyl 2-(dibenzylamino)-2-(2-oxocyclopentyl)acetate 2a}

Syn-2a. Yield 9\% (4.9 mg); yellow oil; $R_{\mathrm{f}}=0.33$ ( $n$ hexane : ethyl acetate $=5: 1) ;{ }^{1} \mathrm{H}$ NMR $\left(500 \mathrm{MHz}, \mathrm{CDCl}_{3}\right) \delta 1.41(\mathrm{t}, J$ $=7.0 \mathrm{~Hz}, 3 \mathrm{H}), 1.59-1.67(\mathrm{~m}, 1 \mathrm{H}), 1.75-1.83(\mathrm{~m}, 1 \mathrm{H}), 1.92-2.07$ $(\mathrm{m}, 2 \mathrm{H}), 2.24-2.30(\mathrm{~m}, 1 \mathrm{H}), 2.35-2.41(\mathrm{~m}, 1 \mathrm{H}), 2.90-2.96(\mathrm{~m}$, $1 \mathrm{H}), 3.19$ (d, $J=11.0 \mathrm{~Hz}, 1 \mathrm{H}), 3.36$ (d, $J=13.7 \mathrm{~Hz}, 2 \mathrm{H}), 3.92(\mathrm{~d}, J$ $=13.7 \mathrm{~Hz}, 2 \mathrm{H}), 4.28-4.41(\mathrm{~m}, 2 \mathrm{H}), 7.23-7.39(\mathrm{~m}, 10 \mathrm{H}) ;{ }^{13} \mathrm{C}$ NMR $\left(126 \mathrm{MHz}, \mathrm{CDCl}_{3}\right) \delta$ 14.6, 20.5, 27.8, 37.9, 49.4 54.3, 60.4, 61.0, $127.2,128.3,128.8,139.0,170.3,218.9$; IR (neat) 3029, 2931, 1731, 1494, 1452, 1371, 1311, 1030, 966, 743, 698, $598 \mathrm{~cm}^{-1}$; HRMS (EI) calcd for $\mathrm{C}_{23} \mathrm{H}_{27} \mathrm{NO}_{3}(\mathrm{M})^{+}$365.1991, found 365.2003.

\section{Ethyl 2-(dibenzylamino)-2-(2-oxocycloheptyl)acetate 2c}

Syn-2c. Yield 40\% (23.9 mg); yellow oil; $R_{\mathrm{f}}=0.31$ ( $n$ hexane : ethyl acetate $=5: 1) ;{ }^{1} \mathrm{H}$ NMR $\left(400 \mathrm{MHz}, \mathrm{CDCl}_{3}\right) \delta 0.85-$ $0.99(\mathrm{~m}, 1 \mathrm{H}), 1.09-1.18(\mathrm{~m}, 1 \mathrm{H}), 1.21-1.40$ (m, 5H including triplet at $1.38 \mathrm{ppm}, J=7.3 \mathrm{~Hz}, 3 \mathrm{H}), 1.60-1.94(\mathrm{~m}, 4 \mathrm{H}), 2.23-2.32$ $(\mathrm{m}, 1 \mathrm{H}), 2.53-2.60(\mathrm{~m}, 1 \mathrm{H}), 3.08-3.15(\mathrm{~m}, 1 \mathrm{H}), 3.45(\mathrm{~d}, J=$ $13.8 \mathrm{~Hz}, 2 \mathrm{H}), 3.48$ (d, $J=11.0 \mathrm{~Hz}, 1 \mathrm{H}), 3.85$ (d, $J=13.8 \mathrm{~Hz}, 2 \mathrm{H})$, 4.16-4.35 (m, 2H), 7.21-7.34 (m, 10H); ${ }^{13} \mathrm{C}$ NMR (100 MHz, $\left.\mathrm{CDCl}_{3}\right) \delta 14.8,23.3,27.6,28.7,29.3,43.6,50.4$, 55.6, 60.4, 61.9, $127.2,128.4,129.3,139.2,171.7,215.0$; IR (neat) 3029, 2929, 2853, 1453, 1375,1183, 1134, 1024, 938, 750, $700 \mathrm{~cm}^{-1}$; HRMS (EI) calcd for $\mathrm{C}_{25} \mathrm{H}_{31} \mathrm{NO}_{3}(\mathrm{M})^{+}$393.2304, found 393.2327.

Anti-2c. Yield $31 \%\left(13.0 \mathrm{mg}\right.$ ); yellow oil; $R_{\mathrm{f}}=0.51$ ( $n$ hexane : ethyl acetate $=5: 1) ;{ }^{1} \mathrm{H}$ NMR $\left(500 \mathrm{MHz} \mathrm{CDCl}_{3}\right) \delta 1.03-$ $1.40(\mathrm{~m}, 6 \mathrm{H}$ including a triplet at $1.38 \mathrm{ppm}, J=7.0 \mathrm{~Hz}, 3 \mathrm{H})$, 1.56-1.79 (m, 5H), 2.02-2.15 (m, 2H), 3.13-3.21 (m, 1H), 3.28 (d, $J=13.3 \mathrm{~Hz}, 2 \mathrm{H}), 3.60(\mathrm{~d}, J=11.4 \mathrm{~Hz}, 1 \mathrm{H}), 3.96(\mathrm{~d}, J=13.3 \mathrm{~Hz}$, $2 \mathrm{H}), 4.21-4.37$ (m, 2H), 7.18-7.32 (m, 10H); ${ }^{13} \mathrm{C}$ NMR (126 MHz, $\left.\mathrm{CDCl}_{3}\right) \delta 14.8,25.7,26.8,28.0,29.6,41.2,52.3$, 54.8, 60.5, 63.6, 127.2, 128.3, 129.4, 139.1, 169.9, 212.7; IR (neat) 3027, 2933, 2855, 1722, 1494, 1451, 1370, 1326, 1236, 1170, 1025, $750 \mathrm{~cm}^{-1}$; HRMS (EI) calcd for $\mathrm{C}_{25} \mathrm{H}_{31} \mathrm{NO}_{3}(\mathrm{M})^{+}$393.2304, found 393.2324.

\section{Ethyl 2-(dibenzylamino)-2-(2-oxocyclooctyl)acetate 2d}

Syn-2d. Yield 41\% (24.5 mg); yellow oil; $R_{\mathrm{f}}=0.32$ ( $n$ hexane : ethyl acetate $=5: 1) ;{ }^{1} \mathrm{H}$ NMR $\left(400 \mathrm{MHz} \mathrm{CDCl}_{3}\right) \delta 0.92-$ $0.95(\mathrm{~m}, 1 \mathrm{H}), 1.08-1.13(\mathrm{~m}, 1 \mathrm{H}), 1.17-1.26(\mathrm{~m}, 1 \mathrm{H}), 1.37-1.57$ $(\mathrm{m}, 9 \mathrm{H}$ including triplet at $1.38 \mathrm{ppm}, J=7.0 \mathrm{~Hz}, 3 \mathrm{H}), 1.69-1.73$ $(\mathrm{m}, 1 \mathrm{H}), 2.00-2.04(\mathrm{~m}, 1 \mathrm{H}), 2.14(\mathrm{~d}, \mathrm{~d}, \mathrm{~d}, J=2.8 \mathrm{~Hz}, 7.6 \mathrm{~Hz}$, $15.1 \mathrm{~Hz}, 1 \mathrm{H}), 3.19$ (d,t, $J=3.7 \mathrm{~Hz}, 10.6 \mathrm{~Hz}, 1 \mathrm{H}), 3.46(\mathrm{~d}, J=$ $13.4 \mathrm{~Hz}, 2 \mathrm{H}), 3.58$ (d, $J=10.6 \mathrm{~Hz}, 1 \mathrm{H}), 3.85$ (d, $J=13.4 \mathrm{~Hz}, 2 \mathrm{H})$, 4.17-4.32 (m, 2H), 7.23-7.34 (m, 10H); ${ }^{13} \mathrm{C}$ NMR (100 MHz, $\left.\mathrm{CDCl}_{3}\right) \delta 14.7,22.8,24.4,25.1,28.5,31.8$ 43.9, 48.0, 55.6, 60.4, $63.8,127.2,128.3,129.3,139.0,171.8,219.2$; IR (neat) 2931, 2854, 1719, 1696, 1647,1454, 1027, 748, $698 \mathrm{~cm}^{-1}$; HRMS (EI) calcd for $\mathrm{C}_{26} \mathrm{H}_{33} \mathrm{NO}_{3}(\mathrm{M})^{+}$407.2460, found 407.2457 .

Anti-2d. Yield 27\% (16.3 mg); yellow oil; $R_{\mathrm{f}}=0.51$ ( $n$ hexane : ethyl acetate $=5: 1) ;{ }^{1} \mathrm{H}$ NMR $\left(500 \mathrm{MHz}, \mathrm{CDCl}_{3}\right) \delta 1.26$ $1.85(\mathrm{~m}, 13 \mathrm{H}$ including a triplet at $1.38 \mathrm{ppm}, J=7.0 \mathrm{~Hz}, 3 \mathrm{H})$, 2.04-2.19 (m, 2H), 3.25 (d, $J=13.7 \mathrm{~Hz}, 2 \mathrm{H}), 3.27$ (d, $J=10.8 \mathrm{~Hz}$, $1 \mathrm{H}), 3.56(\mathrm{~d}, J=11.6 \mathrm{~Hz}, 1 \mathrm{H}), 3.98$ (d, $J=13.7 \mathrm{~Hz}, 2 \mathrm{H}), 4.21-4.37$ $(\mathrm{m}, 2 \mathrm{H}), 7.22-7.34(\mathrm{~m}, 10 \mathrm{H}) ;{ }^{13} \mathrm{C} \mathrm{NMR}\left(126 \mathrm{MHz}, \mathrm{CDCl}_{3}\right) \delta 14.7$, 24.6, 25.8, 26.1, 26.7, 28.9, 41.1, 50.4, 54.8, 60.3, 63.8, 127.1,
128.2, 129.2, 138.7, 170.0, 216.0; IR (neat) 2930, 1727, 1702, 1494, 1453, 1154, 1135, 1028, 749, $699 \mathrm{~cm}^{-1}$; HRMS (EI) calcd for $\mathrm{C}_{26} \mathrm{H}_{33} \mathrm{NO}_{3}(\mathrm{M})^{+}$407.2460, found 407.2460.

Synthesis of ethyl 4-cyclohexyl-2-(dibenzylamino)-3-methyl-4oxobutanoate $3 \mathrm{a}$ (general procedure for the addition reaction with acyclic silyl enol ethers)

In a $30 \mathrm{~mL}$ two-necked round-bottomed flask equipped with a magnetic stirring bar, a rubber septum, and an argon balloon was introduced a solution of DDQ $(37.4 \mathrm{mg}, 0.165 \mathrm{mmol})$ in EtCN $(1.0 \mathrm{~mL})$. The solution was cooled to $-55^{\circ} \mathrm{C}$, and to it were added successively solutions of 1-ethoxy-2-dibenzylamino$1^{-}{ }^{t}$ butyldimethylsiloxyethylene $1(59.6 \mathrm{mg}, 0.150 \mathrm{mmol})$ in EtCN

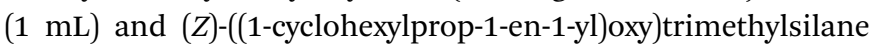
$(63.6 \mathrm{mg}, 0.150 \mathrm{mmol})$ in EtCN $(1 \mathrm{~mL})$. The reaction mixture was allowed to warm to room temperature with stirring for $14 \mathrm{~h}$. The reaction was quenched with $10 \%$ aq. $\mathrm{Na}_{2} \mathrm{CO}_{3}$, and the whole mixture was extracted with ethyl acetate $(10 \mathrm{~mL} \times 3)$. The combined extracts were washed with brine $(15 \mathrm{~mL})$, dried $\left(\mathrm{Na}_{2} \mathrm{SO}_{4}\right)$, and concentrated in vacuo. The crude product was purified by preparative TLC on silica gel $\left(\mathrm{CH}_{2} \mathrm{Cl}_{2}\right.$ : nhexane $=$ $5: 1)$ to give the title compound (syn-3a $(32.5 \mathrm{mg}, 52 \%)$ and anti3a $(9.7 \mathrm{mg}, 15 \%)) .{ }^{10}$

Syn-3a. Yield 52\% (32.7 mg); colorless oil; $R_{\mathrm{f}}=0.35(n$ hexane : ethyl acetate $=5: 1) ;{ }^{1} \mathrm{H}$ NMR $\left(400 \mathrm{MHz}, \mathrm{CDCl}_{3}\right) \delta 0.86(\mathrm{~d}, J$ $=6.9 \mathrm{~Hz}, 3 \mathrm{H}), 0.95-1.37(\mathrm{~m}, 9 \mathrm{H}$ including a triplet at $1.29 \mathrm{ppm}, J$ $=7.1 \mathrm{~Hz}, 3 \mathrm{H}), 1.61-1.82(\mathrm{~m}, 4 \mathrm{H}), 2.14-2.22(\mathrm{~m}, 1 \mathrm{H}), 3.22-3.31$ $(\mathrm{m}, 3 \mathrm{H}$ including a doublet at $3.29 \mathrm{ppm}, J=14.2 \mathrm{~Hz}, 3 \mathrm{H}), 3.59$ $(\mathrm{d}, J=11.0 \mathrm{~Hz}, 1 \mathrm{H}), 3.95$ (d, $J=14.2 \mathrm{~Hz}, 2 \mathrm{H}), 4.11-4.26(\mathrm{~m}, 2 \mathrm{H})$, 7.15-7.26 (m, 10H); $\left.{ }^{13} \mathrm{C} \mathrm{NMR} \mathrm{(100} \mathrm{MHz,} \mathrm{CDCl}_{3}\right) \delta 14.5,15.0$, 25.2, 25.8, 25.9, 28.0, 28.7, 43.7, 51.0, 55.2, 60.2, 65.0, 127.1, 128.2, 128.8, 138.5, 170.4, 213.9; IR (neat) 3027, 2933, 2852, 1728, 1711, 1495, 1450, 1375, 1027, 994, 732, $698 \mathrm{~cm}^{-1}$; HRMS (EI) calcd for $\mathrm{C}_{27} \mathrm{H}_{35} \mathrm{NO}_{3}(\mathrm{M})^{+} 421.2617$, found 421.2623 .

Anti-3a. Yield 15\% (9.5 mg); colorless oil; $R_{\mathrm{f}}=0.56$ ( $n$ hexane : ethyl acetate $=5: 1) ;{ }^{1} \mathrm{H}$ NMR $\left(500 \mathrm{MHz}, \mathrm{CDCl}_{3}\right) \delta 0.97(\mathrm{t}, J$ $=7.1 \mathrm{~Hz}, 3 \mathrm{H}), 1.06(\mathrm{~d}, J=7.3 \mathrm{~Hz}, 3 \mathrm{H}), 1.37(\mathrm{t}, J=7.1 \mathrm{~Hz}, 3 \mathrm{H})$, 2.40-2.58 (m, 2H), 3.01-3.09 (m, 1H), 3.45 (d, $J=13.3 \mathrm{~Hz}, 2 \mathrm{H})$, $3.53(\mathrm{~d}, J=11.0 \mathrm{~Hz}, 1 \mathrm{H}), 3.85(\mathrm{~d}, J=13.3 \mathrm{~Hz}, 2 \mathrm{H}), 4.14-4.32(\mathrm{~m}$, $2 \mathrm{H}), 7.22-7.36(\mathrm{~m}, 10 \mathrm{H}) ;{ }^{13} \mathrm{C} \mathrm{NMR}\left(126 \mathrm{MHz}, \mathrm{CDCl}_{3}\right) \delta 17.6,14.6$, 14.8, 34.9, 45.1, 55.2, 60.4, 62.7, 127.2, 128.3, 129.2, 138.9, 171.9, 214.0; IR (neat) 2931, 2851, 1725, 1495, 1452, 1368, 1027, 914, $698 \mathrm{~cm}^{-1}$; HRMS (EI) calcd for $\mathrm{C}_{27} \mathrm{H}_{35} \mathrm{NO}_{3}(\mathrm{M})^{+} 421.2617$, found 421.2619 .

\section{Ethyl 2-(dibenzylamino)-3-methyl-4-oxo-4-phenylbutanoate $3 b^{10}$}

A mixture of syn- and anti-isomers $(35.4 \mathrm{mg}, 57 \%$, syn $:$ anti $=$ $57: 43$, ratio determined by ${ }^{1} \mathrm{H}$ NMR). Yellow oil; $R_{\mathrm{f}}=0.52$ (nhexane : ethyl acetate $=5: 1$ ); IR (neat) 3061, 2979, 1724, 1683, 1495, 1027, 748, $698 \mathrm{~cm}^{-1}$; HRMS (EI) calcd for $\mathrm{C}_{27} \mathrm{H}_{29} \mathrm{NO}_{3}(\mathrm{M})^{+}$415.2147, found 415.2136.

Syn-3b. ${ }^{1} \mathrm{H}$ NMR $\left(400 \mathrm{MHz}, \mathrm{CDCl}_{3}\right) \delta 1.16(\mathrm{~d}, J=7.3 \mathrm{~Hz}, 3 \mathrm{H})$, $1.29(\mathrm{t}, J=7.1 \mathrm{~Hz}, 3 \mathrm{H}), 3.56$ (d, $J=13.3 \mathrm{~Hz}, 2 \mathrm{H}), 3.79$ (d, $J=$ $10.5 \mathrm{~Hz}, 1 \mathrm{H}), 3.86-3.97$ (m, $3 \mathrm{H}$ including a doublet at $3.96 \mathrm{ppm}$, $J=13.3 \mathrm{~Hz}, 2 \mathrm{H}), 4.10-4.38(\mathrm{~m}, 2 \mathrm{H}), 7.02-7.58(\mathrm{~m}, 13 \mathrm{H}), 7.87-$ $7.96(\mathrm{~m}, 2 \mathrm{H}) ;{ }^{13} \mathrm{C}$ NMR $\left(100 \mathrm{MHz}, \mathrm{CDCl}_{3}\right) \delta 14.5,15.8,40.4,55.3$, 
$60.3,63.4,60.4,62.7,126.9,127.9,128.2,128.5,128.8,138.2$, 136.2, 138.9, 171.7, 203.3.

Anti-3b. ${ }^{1} \mathrm{H}$ NMR $\left(500 \mathrm{MHz}, \mathrm{CDCl}_{3}\right) \delta 1.08(\mathrm{~d}, J=6.4 \mathrm{~Hz}, 3 \mathrm{H})$, $1.39(\mathrm{t}, J=7.1 \mathrm{~Hz}, 3 \mathrm{H}), 3.34(\mathrm{~d}, J=13.8 \mathrm{~Hz}, 2 \mathrm{H}), 3.86-3.94(\mathrm{~m}$, $3 \mathrm{H}), 4.10-4.38$ (m, 3H), 7.02-7.58 (m, 13H), 7.87-7.96 (m, 2H); ${ }^{13} \mathrm{C}$ NMR $\left(126 \mathrm{MHz}, \mathrm{CDCl}_{3}\right) \delta 14.7,15.4,39.5,55.5,60.2,65.5$, 127.2 , 128.1, 128.3, 128.7, 129.2, 132.9, 137.3, 138.5, 170.4, 201.0.

\section{Synthesis of (E)-N,N-dibenzyl-2-[(tert-butyldimethylsilyl)oxy]- 2-phenylethen-1-amine 5}

Under an argon atmosphere, to a solution of 2-(dibenzylamino)1-phenylethanone $7(4.00 \mathrm{~g}, 12.7 \mathrm{mmol})$ in $\mathrm{CH}_{2} \mathrm{Cl}_{2}(19.0 \mathrm{~mL})$ were successively added triethylamine $(3.54 \mathrm{~mL}, 25.4 \mathrm{mmol})$ and TBSOTf ( $3.50 \mathrm{~mL}, 15.2 \mathrm{mmol})$ at $0{ }^{\circ} \mathrm{C}$. The reaction mixture was allowed to warm to ambient temperature with stirring for $21 \mathrm{~h}$. It was quenched with sat. aq. $\mathrm{NaHCO}_{3}(20 \mathrm{~mL})$. The layers were separated, and the aqueous layer was extracted with $\mathrm{CH}_{2} \mathrm{Cl}_{2}(30 \mathrm{~mL} \times 3)$. The combined extracts were washed with brine $(20 \mathrm{~mL})$, dried over anhydrous $\mathrm{Na}_{2} \mathrm{SO}_{4}$, and concentrated in vacuo. The crude product was purified by column chromatography on silica gel ( $n$ hexane : AcOEt : $\mathrm{Et}_{3} \mathrm{~N}=20: 1: 1.1$ ) to give the title amino silyl enol ether $5(89 \%, 4.84 \mathrm{~g}, E: Z=$ 89 : 11).

Yield $89 \%$ (4.84 g); yellow solid; $\mathrm{mp}=55-56{ }^{\circ} \mathrm{C} ; R_{\mathrm{f}}=0.75$ ( $n$ hexane : ethyl acetate $=5: 1) ;{ }^{1} \mathrm{H}$ NMR $\left(400 \mathrm{MHz}, \mathrm{CDCl}_{3}\right.$ ): $\delta 0.15(\mathrm{~s}, 6 \mathrm{H}), 1.11(\mathrm{~s}, 9 \mathrm{H}), 4.33(\mathrm{~s}, 4 \mathrm{H}), 5.82(\mathrm{~s}, 1 \mathrm{H}), 7.32-7.45$ $(\mathrm{m}, 15 \mathrm{H}) ;{ }^{13} \mathrm{C}$ NMR $\left(100 \mathrm{MHz}, \mathrm{CDCl}_{3}\right): \delta-3.9,18.3,26.0,55.8$, $123.4,124.5,125.5,126.9,127.9$, 128.2, 128.7, 133.1, 139.0, 140.4; IR (neat): 3059, 3031, 2926, 2854, 1649, 1453, 1349, 1257, 1162, 1058, 1024, 923, 749, $703 \mathrm{~cm}^{-1}$. HRMS (EI) calcd for $\mathrm{C}_{28} \mathrm{H}_{35} \mathrm{NOSi}(\mathrm{M})^{+}$429.2488, found 429.2500.

Synthesis of methyl 3-(dibenzylamino)-2,2-dimethyl-4-oxo-4phenylbutanoate $8 \mathrm{a}$ (general procedure for the reaction of the amino silyl enol ether with ketene silyl acetals)

Under an argon atmosphere, to $\mathrm{N}$-bromosuccinimide $(19.6 \mathrm{mg}$, $0.11 \mathrm{mmol}$ ) were successively added a solution of the amino silyl enol ether 5 ( $42.9 \mathrm{mg}, 0.10 \mathrm{mmol})$ in $\mathrm{EtCN}(1.0 \mathrm{~mL}), \mathrm{BF}_{3} \cdot \mathrm{Et}_{2} \mathrm{O}$ $(0.025 \mathrm{~mL}, 0.20 \mathrm{mmol})$, and a solution of [(1-methoxy-2methylprop-1-en-1-yl)oxy]trimethylsilane (26.1 mg, $0.10 \mathrm{mmol}$ ) in EtCN $(1.0 \mathrm{~mL})$ at room temperature. The reaction mixture was stirred for $16 \mathrm{~h}$. It was quenched with sat. aq. $\mathrm{NaHCO}_{3}(10$ $\mathrm{mL})$. The layers were separated, and the aqueous layer was extracted with ethyl acetate $(30 \mathrm{~mL} \times 3)$. The combined organic extracts were washed with brine $(5 \mathrm{~mL})$, dried over anhydrous $\mathrm{Na}_{2} \mathrm{SO}_{4}$, and concentrated in vacuo. The crude product was purified by preparative TLC on silica gel (developed twice with nhexane : AcOEt $=6: 1$ ) to give methyl 3-(dibenzylamino)-2,2dimethyl-4-oxo-4-phenylbutanoate 8a (80\%, $33.4 \mathrm{mg})$.

Yield $80 \%$ (33.4 mg); white solid; $\mathrm{mp}=73-74{ }^{\circ} \mathrm{C} ; R_{\mathrm{f}}=0.48$ (nhexane : ethyl acetate $=5: 1) ;{ }^{1} \mathrm{H}$ NMR $\left(400 \mathrm{MHz}, \mathrm{CDCl}_{3}\right.$ ) $\delta 1.11(\mathrm{~s}, 3 \mathrm{H}), 1.44(\mathrm{~s}, 3 \mathrm{H}), 3.26(\mathrm{~d}, J=14.4 \mathrm{~Hz}, 2 \mathrm{H}), 3.49(\mathrm{~s}, 3 \mathrm{H})$, $4.01(\mathrm{~d}, J=14.4 \mathrm{~Hz}, 2 \mathrm{H}), 4.68(\mathrm{~s}, 1 \mathrm{H}), 7.23-7.37$ (m, 10H), 7.527.57 (m, 2H), 7.60-7.65 (m, 1H), 7.87-7.90 (m, 2H); ${ }^{13} \mathrm{C}$ NMR $\left(100 \mathrm{MHz}, \mathrm{CDCl}_{3}\right): \delta$ 21.6, 25.6, 46.6, 51.8, 56.9, 64.4, 127.2,
128.2, 128.3, 128.7, 129.0, 132.8, 139.4, 141.2, 177.7, 202.1; IR (neat) 2949, 1729, 1678, 1451, 1268, 1145, 970, 745, $700 \mathrm{~cm}^{-1}$; HRMS (EI) calcd for $\mathrm{C}_{26} \mathrm{H}_{26} \mathrm{NO}_{2}\left(\mathrm{M}-\mathrm{CH}_{3} \mathrm{O}\right)^{+} 384.1958$, found 384.1961.

Ethyl 3-(dibenzylamino)-2,2-dimethyl-4-oxo-4phenylbutanoate $8 \mathrm{~b}$

Yield $82 \%$ (35.1 mg); white solid; $\mathrm{mp}=65-67{ }^{\circ} \mathrm{C} ; R_{\mathrm{f}}=0.41$ (nhexane : ethyl acetate $=5: 1) ;{ }^{1} \mathrm{H}$ NMR $\left(400 \mathrm{MHz}, \mathrm{CDCl}_{3}\right.$ ) $\delta 1.06(\mathrm{t}, J=7.3 \mathrm{~Hz}, 3 \mathrm{H}), 1.08(\mathrm{~s}, 3 \mathrm{H}), 1.46(\mathrm{~s}, 3 \mathrm{H}), 3.26(\mathrm{~d}, J=$ $14.2 \mathrm{~Hz}, 2 \mathrm{H}$ ), 3.90 (dq, $J=7.3,10.5 \mathrm{~Hz}, 1 \mathrm{H}), 3.97-4.05(\mathrm{~m}, 3 \mathrm{H}$, including doublet at $4.01 \mathrm{ppm} J=14.2 \mathrm{~Hz}, 2 \mathrm{H}), 4.67(\mathrm{~s}, 1 \mathrm{H})$, 7.22-7.35 (m, 10H), 7.52-7.56 (m, 2H), 7.59-7.64 (m, 1H), 7.88$7.90(\mathrm{~m}, 2 \mathrm{H}) ;{ }^{13} \mathrm{C}$ NMR $\left(100 \mathrm{MHz}, \mathrm{CDCl}_{3}\right) \delta 13.9,21.3,25.9,46.5$, 56.8, 60.5, 64.4, 127.2, 128.2, 128.4, 128.6, 129.0, 132.8, 139.4, 141.3, 177.3, 202.2; IR (neat): 3061, 3028, 2980, 2842, 1735, 1675, 1455, 1274, 1152, 968, 748, $699 \mathrm{~cm}^{-1}$; HRMS (EI) calcd for $\mathrm{C}_{26} \mathrm{H}_{26} \mathrm{NO}_{2}\left(\mathrm{M}-\mathrm{C}_{2} \mathrm{H}_{5} \mathrm{O}\right)^{+}$384.1958, found 384.1957.

\section{Isopropyl 3-(dibenzylamino)-2,2-dimethyl-4-oxo-4- phenylbutanoate $8 \mathrm{c}$}

Yield $73 \%$ (32.6 mg); white solid $\mathrm{mp}=112-113{ }^{\circ} \mathrm{C} ; R_{\mathrm{f}}=0.55$ ( $n$ hexane : ethyl acetate $=5: 1) ;{ }^{1} \mathrm{H}$ NMR $\left(400 \mathrm{MHz}, \mathrm{CDCl}_{3}\right.$ ) $\delta 1.01(\mathrm{~d}, J=6.4 \mathrm{~Hz}, 3 \mathrm{H}), 1.02(\mathrm{~s}, 3 \mathrm{H}), 1.08(\mathrm{~d}, J=6.4 \mathrm{~Hz}, 3 \mathrm{H})$, $1.49(\mathrm{~s}, 3 \mathrm{H}), 3.26$ (d, $J=14.2 \mathrm{~Hz}, 2 \mathrm{H}), 4.00(\mathrm{~d}, J=14.2 \mathrm{~Hz}, 2 \mathrm{H})$, $4.64(\mathrm{~s}, 1 \mathrm{H}), 4.84$ (sept, $J=6.4 \mathrm{~Hz}, 1 \mathrm{H}), 7.22-7.36(\mathrm{~m}, 10 \mathrm{H}), 7.52-$ $7.56(\mathrm{~m}, 2 \mathrm{H}), 7.60-7.64(\mathrm{~m}, 1 \mathrm{H}), 7.88-7.91(\mathrm{~m}, 2 \mathrm{H}) ;{ }^{13} \mathrm{C} \mathrm{NMR}$ $\left(100 \mathrm{MHz}, \mathrm{CDCl}_{3}\right) \delta 20.7,21.3,21.6,26.5,46.4,56.8,64.5,67.8$, 127.2, 128.2, 128.4, 128.6, 129.0, 132.7, 139.4, 141.4, 176.9, 202.3; IR (neat): 3063, 3030, 2981, 2855, 1711, 1676, 1455, 1281, 1165, 1107, 970, 749, $694 \mathrm{~cm}^{-1}$; HRMS (EI): calcd for $\mathrm{C}_{26} \mathrm{H}_{26} \mathrm{NO}_{2}$ $\left(\mathrm{M}-\mathrm{C}_{3} \mathrm{H}_{7} \mathrm{O}\right)^{+}$384.1958, found 384.1957.

\section{tert-Butyl 3-(dibenzylamino)-2,2-dimethyl-4-oxo-4- phenylbutanoate 8d}

Yield 57\% (26.3 mg); white solid; $\mathrm{mp}=115-117^{\circ} \mathrm{C} ; R_{\mathrm{f}}=0.58$ ( $n$ hexane : ethyl acetate $=5: 1) ;{ }^{1} \mathrm{H}$ NMR $\left(400 \mathrm{MHz}, \mathrm{CDCl}_{3}\right.$ ) $\delta 0.98(\mathrm{~s}, 3 \mathrm{H}), 1.27(\mathrm{~s}, 9 \mathrm{H}), 1.47(\mathrm{~s}, 3 \mathrm{H}), 3.28(\mathrm{~d}, J=14.2 \mathrm{~Hz}, 2 \mathrm{H})$, $3.98(\mathrm{~d}, J=14.2 \mathrm{~Hz}, 2 \mathrm{H}), 4.62(\mathrm{~s}, 1 \mathrm{H}), 7.22-7.37$ (m, 10H), 7.51$7.56(\mathrm{~m}, 2 \mathrm{H}), 7.59-7.63(\mathrm{~m}, 1 \mathrm{H}), 7.88-7.90(\mathrm{~m}, 2 \mathrm{H}) ;{ }^{13} \mathrm{C}$ NMR $\left(100 \mathrm{MHz}, \mathrm{CDCl}_{3}\right) \delta 20.6,26.8,27.8,46.9,56.7,64.6,80.5,127.2$, 128.2, 128.5, 128.6, 129.0, 132.6, 139.4, 141.5, 176.6, 202.4; IR (neat): 2976, 1678, 1456, 1247, 1146, 968, 848, $698 \mathrm{~cm}^{-1}$; HRMS (EI) calcd for $\mathrm{C}_{30} \mathrm{H}_{35} \mathrm{NO}_{3}(\mathrm{M})^{+} 457.2617$, found 457.2607 .

\section{Methyl 3-(dibenzylamino)-2,2-dimethoxy-4-oxo-4- phenylbutanoate $8 \mathrm{e}$}

Yield $73 \%$ (32.6 mg); colorless oil; $R_{\mathrm{f}}=0.38$ (nhexane : ethyl acetate $=5: 1) ;{ }^{1} \mathrm{H} \mathrm{NMR}\left(400 \mathrm{MHz}, \mathrm{CDCl}_{3}\right) \delta 3.17(\mathrm{~s}, 3 \mathrm{H}), 3.27(\mathrm{~s}$, $3 \mathrm{H}), 3.65$ (d, $J=13.7 \mathrm{~Hz}, 2 \mathrm{H}), 3.71(\mathrm{~s}, 3 \mathrm{H}), 4.26$ (d, $J=13.7 \mathrm{~Hz}$, 2H), 4.92 (s, 1H), 7.17-7.28 (m, 10H), 7.39-7.43 (m, 2H), 7.53$7.57(\mathrm{~m}, 1 \mathrm{H}), 7.76-7.79(\mathrm{~m}, 2 \mathrm{H}) ;{ }^{13} \mathrm{C} \mathrm{NMR}\left(100 \mathrm{MHz}, \mathrm{CDCl}_{3}\right)$ $\delta$ 51.0, 51.2, 52.5, 55.5, 63.4, 103.8, 127.0, 128.0, 128.2, 128.8, 129.4, 132.9, 139.1, 139.6, 168.4, 200.9; IR (neat) 2950, 2821, 
1757, 1681, 1547, 1214, 1073, 749, $696 \mathrm{~cm}^{-1}$; HRMS (EI) calcd for $\mathrm{C}_{27} \mathrm{H}_{29} \mathrm{NO}_{5}(\mathrm{M})^{+}$447.2046, found 447.2047.

Ethyl 3-(dibenzylamino)-2,2-diethoxy-4-oxo-4phenylbutanoate 2-8f

Yield 70\% (34.1 mg); white solid $\mathrm{mp}=84-85{ }^{\circ} \mathrm{C} ; R_{\mathrm{f}}=0.46$ ( $n$ hexane : ethyl acetate $=5: 1$ ); $\delta{ }^{1} \mathrm{H}$ NMR (400 MHz, $\mathrm{CDCl}_{3}$ ): $\delta 1.06(\mathrm{t}, J=6.9 \mathrm{~Hz}, 6 \mathrm{H}), 1.20(\mathrm{t}, J=7.3 \mathrm{~Hz}, 3 \mathrm{H}), 3.30-3.40(\mathrm{~m}$, $2 \mathrm{H}), 3.48(\mathrm{dq}, J=6.9,9.1 \mathrm{~Hz}, 1 \mathrm{H}), 3.61(\mathrm{dq}, J=6.9,9.1 \mathrm{~Hz}, 1 \mathrm{H})$, 3.72 (d, $J=13.7 \mathrm{~Hz}, 2 \mathrm{H}), 4.05$ (dq, $J=7.3,10.5 \mathrm{~Hz}, 1 \mathrm{H}), 4.23-$ $4.32(\mathrm{~m}, 3 \mathrm{H}$, including doublet at $4.27 \mathrm{ppm} J=13.7 \mathrm{~Hz}, 2 \mathrm{H})$, 4.89 (s, 1H), 7.20-7.27 (m, 10H), 7.35-7.39 (m, 2H), 7.49-7.53 $(\mathrm{m}, 1 \mathrm{H}), 7.72-7.74(\mathrm{~m}, 2 \mathrm{H}) ;{ }^{13} \mathrm{C} \mathrm{NMR}\left(100 \mathrm{MHz}, \mathrm{CDCl}_{3}\right) \delta 13.9$, 14.8, 15.1, 55.5, 58.9, 59.2, 61.5, 64.7, 103.0, 126.8, 127.8, 127.9, 128.8, 129.5, 132.4, 139.5, 139.9, 168.2, 201.5; IR (neat) 2979, 1750, 1682, 1559, 1452, 1249, 1122, 1062, 748, $696 \mathrm{~cm}^{-1}$; HRMS (EI) calcd for $\mathrm{C}_{28} \mathrm{H}_{30} \mathrm{NO}_{4}\left(\mathrm{M}-\mathrm{C}_{2} \mathrm{H}_{5} \mathrm{O}\right)^{+} 444.2169$, found 444.2170.

\section{S-tert-Butyl 3-(dibenzylamino)-4-oxo-4-phenylbutanethioate 8g}

Yield 63\% (30.0 mg); colorless oil; $R_{\mathrm{f}}=0.64$ (nhexane : ethyl acetate $=5: 1) ;{ }^{1} \mathrm{H} \mathrm{NMR}\left(400 \mathrm{MHz}, \mathrm{CDCl}_{3}\right) \delta 1.45(\mathrm{~s}, 9 \mathrm{H}), 2.97$ (dd, $J=3.6,15.5 \mathrm{~Hz}, 1 \mathrm{H}), 3.30$ (dd, $J=8.7,15.5 \mathrm{~Hz}, 1 \mathrm{H}), 3.46$ (d, $J=13.3 \mathrm{~Hz}, 2 \mathrm{H}), 3.66(\mathrm{~d}, J=13.3 \mathrm{~Hz}, 2 \mathrm{H}), 4.83$ (dd, $J=3.6$, $8.7 \mathrm{~Hz}, 1 \mathrm{H}), 7.10-7.13(\mathrm{~m}, 4 \mathrm{H}), 7.23-7.32(\mathrm{~m}, 8 \mathrm{H}), 7.47-7.50(\mathrm{~m}$, 1H), 7.51-7.56 (m, 2H); $\left.{ }^{13} \mathrm{C} \mathrm{NMR} \mathrm{(100} \mathrm{MHz,} \mathrm{CDCl}_{3}\right) \delta 29.8,38.3$, 48.2, 54.5, 59.0, 127.3, 128.1, 128.2, 129.0, 129.3, 132.7, 136.4, 138.5, 198.6, 198.9; IR (neat) 3061, 3028, 2980, 2842, 1735, 1675, 1455, 1274, 1152, 968, 748, $699 \mathrm{~cm}^{-1}$; HRMS (EI) calcd for $\mathrm{C}_{28} \mathrm{H}_{31} \mathrm{NO}_{2} \mathrm{~S}(\mathrm{M})^{+}$445.2076, found 445.2077.

Synthesis of 2-(dibenzylamino)-1-phenyl-2-[1-

(triisopropylsilyl)-1H-indol-3-yl] ethenone 9a (general procedure for the reaction of the amino silyl enol ether with indoles)

Under an argon atmosphere, to a solution of $\mathrm{N}$-bromosuccinimide (39.2 $\mathrm{mg}, 0.22 \mathrm{mmol})$ in EtCN $(1.0 \mathrm{~mL})$ were successively added a solution of the amino silyl enol ether 5 (85.9 $\mathrm{mg}, 0.20$ $\mathrm{mmol})$ in $\mathrm{EtCN}(1.0 \mathrm{~mL}), \mathrm{BF}_{3} \cdot \mathrm{Et}_{2} \mathrm{O}(0.025 \mathrm{~mL}, 0.20 \mathrm{mmol})$, and a solution of 1-(triisopropylsilyl)-1H-indole (43.1 mg, 0.22 $\mathrm{mmol}$ ) in EtCN at $0{ }^{\circ} \mathrm{C}$. The reaction mixture was allowed to warm to ambient temperature with stirring for $5 \mathrm{~h}$. It was quenched with sat. aq. $\mathrm{NaHCO}_{3}(10 \mathrm{~mL})$. The layers were separated, and the aqueous layer was extracted with ethyl acetate $(30 \mathrm{~mL} \times 3)$. The combined organic extracts were washed with brine, dried over anhydrous $\mathrm{Na}_{2} \mathrm{SO}_{4}$, and concentrated in vacuo. The crude product was purified by preparative TLC on silica gel (developed twice with $n$ hexane $:$ AcOEt $=6: 1$ ) to give 2-(dibenzylamino)-1-phenyl-2-[1-(triisopropylsilyl)-1 $H^{-}$ indol-3-yl] ethanone 9a (77\%, $90.7 \mathrm{mg})$.

Yield 77\% (90.7 mg); yellow oil; $R_{\mathrm{f}}=0.61$ ( $n$ hexane : ethyl acetate $=6: 1) ;{ }^{1} \mathrm{H} \mathrm{NMR}\left(400 \mathrm{MHz}, \mathrm{CDCl}_{3}\right) \delta 0.95(\mathrm{~d}, J=7.3 \mathrm{~Hz}$, 9H), 0.99 (d, $J=7.3 \mathrm{~Hz}, 9 \mathrm{H}), 1.55$ (sept, $J=7.3 \mathrm{~Hz}, 3 \mathrm{H}), 3.94$ (d, $J$ $=13.7 \mathrm{~Hz}, 2 \mathrm{H}), 4.02(\mathrm{~d}, J=13.7 \mathrm{~Hz}, 2 \mathrm{H}), 5.78(\mathrm{~s}, 1 \mathrm{H}), 6.91(\mathrm{~s}$, 1H), 7.16-7.30 (m, 14H), 7.34-7.37 (m, 1H), 7.44-7.46 (m, 1H), 7.65-7.68 (m, 3H); ${ }^{13} \mathrm{C} \mathrm{NMR}\left(100 \mathrm{MHz}, \mathrm{CDCl}_{3}\right) \delta 12.6,17.8,17.9$, 54.8, 59.8, 113.0, 113.9, 119.5, 119.9, 121.9, 126.7, 128.0, 128.1,
128.2, 129.1, 130.6, 132.5, 137.0, 140.6, 141.4, 201.7; IR (neat) 3060, 3026, 2948, 2868, 1685, 1494, 1449, 1165, 1144, 1074, 967, 882, 746, 696, $664 \mathrm{~cm}^{-1}$; HRMS (EI) calcd for $\mathrm{C}_{39} \mathrm{H}_{46} \mathrm{~N}_{2} \mathrm{OSi}(\mathrm{M})^{+}$ 586.3380 , found 586.3379 .

2-(Dibenzylamino)-2-[5-nitro-1-(triisopropylsilyl)-1H-indol-3yl]-1-phenylethan-1-one $9 \mathrm{~b}$

Yield 13\% (15.9 mg); yellow oil; $R_{\mathrm{f}}=0.43$ (nhexane : ethyl acetate $=6: 1) ;{ }^{1} \mathrm{H}$ NMR $\left(400 \mathrm{MHz}, \mathrm{CDCl}_{3}\right) \delta 0.92-1.00(\mathrm{~m}, 18 \mathrm{H})$, 1.49-1.60 (m, 3H), 3.96 (dd, $J=13.8,29.1 \mathrm{~Hz}, 4 \mathrm{H}), 5.80(\mathrm{~s}, 1 \mathrm{H})$, 7.04-8.65 (m, 19H); ${ }^{13} \mathrm{C}$ NMR $\left(100 \mathrm{MHz}, \mathrm{CDCl}_{3}\right) \delta 12.5,17.7$, 17.8, 54.8, 59.0, 113.7, 115.5, 116.8, 117.6, 127.1, 128.1, 128.4, $128.5,129.1,130.3,133.0,135.2$, 136.9, 140.0, 141.9, 144.5, 201.2; IR (neat) 3064, 3025, 2945, 2866, 1683, 1508, 1447, 1338, 1264, 1206, 1136, 970, 808, 688, $659 \mathrm{~cm}^{-1}$; HRMS (EI) calcd for $\mathrm{C}_{30} \mathrm{H}_{39} \mathrm{~N}_{3} \mathrm{O}_{3} \mathrm{Si}(\mathrm{M})^{+}$631.3230, found 631.3239.

\section{2-(Dibenzylamino)-2-[5-methoxy-1-(triisopropylsilyl)-1H-indol-} 3-yl]-1-phenylethanone 9c

Yield $67 \%$ (82.7 mg); yellow oil; $R_{\mathrm{f}}=0.55$ (nhexane : ethyl acetate $=6: 1) ;{ }^{1} \mathrm{H}$ NMR $\left(400 \mathrm{MHz}, \mathrm{CDCl}_{3}\right) \delta 0.92(\mathrm{~d}, J=7.6 \mathrm{~Hz}$, 9H), 0.96 (d, $J=7.6 \mathrm{~Hz}, 9 \mathrm{H}), 1.50$ (sept, $J=7.6 \mathrm{~Hz}, 3 \mathrm{H}$ ), 3.89 (s, $3 \mathrm{H}), 3.99(\mathrm{~s}, 4 \mathrm{H}), 5.73(\mathrm{~s}, 1 \mathrm{H}), 6.80-6.83(\mathrm{~m}, 2 \mathrm{H}), 7.05-7.07(\mathrm{~m}$, $1 \mathrm{H}), 7.18-7.33(\mathrm{~m}, 13 \mathrm{H}), 7.38-7.40(\mathrm{~m}, 1 \mathrm{H}), 7.68-7.70(\mathrm{~m}, 2 \mathrm{H})$; ${ }^{13} \mathrm{C}$ NMR $\left(100 \mathrm{MHz}, \mathrm{CDCl}_{3}\right) \delta 12.5,17.8,17.9,54.7,55.7,60.0$, 99.9, 100.9, 112.3, 113.1, 114.7, 126.8, 128.1, 128.3, 129.3, 131.1, 132.5, 133.0, 136.2, 137.1, 140.9, 154.2, 202.0; IR (neat) 3061, 3027, 2950, 2867, 1674, 1618, 1485, 1447, 1216, 1038, 1019, 884, 794, 744, 693, 660, $583 \mathrm{~cm}^{-1}$; HRMS (EI) calcd for $\mathrm{C}_{40} \mathrm{H}_{48} \mathrm{~N}_{2} \mathrm{O}_{2} \mathrm{Si}$ $(\mathrm{M})^{+}$616.3485, found 616.3482 .

2-[5-Bromo-1-(triisopropylsilyl)-1H-indol-3-yl]-2(dibenzylamino)-1-phenylethan-1-one 9d

Yield $75 \%$ (100.0 mg); yellow oil; $R_{\mathrm{f}}=0.63$ (nhexane : ethyl acetate $=6: 1) ;{ }^{1} \mathrm{H}$ NMR $\left(400 \mathrm{MHz}, \mathrm{CDCl}_{3}\right) \delta 0.94-0.99(\mathrm{~m}, 18 \mathrm{H})$, $1.53(\mathrm{q}, J=7.6 \mathrm{~Hz}, 3 \mathrm{H}), 3.97$ (dd, $J=13.6,32.2 \mathrm{~Hz}, 4 \mathrm{H}), 5.71$ (s, $1 \mathrm{H}), 6.92(\mathrm{~s}, 1 \mathrm{H}), 7.15-7.48(\mathrm{~m}, 16 \mathrm{H}), 7.68-7.79(\mathrm{~m}, 2 \mathrm{H}) ;{ }^{13} \mathrm{C}$ NMR $\left(100 \mathrm{MHz}, \mathrm{CDCl}_{3}\right) \delta 12.6,17.9,18.0,54.8,59.5,113.0$, $113.5,115.4,122.4,124.9,127.1,128.2$, 128.3, 128.5, 129.3, 132.6, 132.8, 133.6, 137.0, 140.1, 140.5, 201.5; IR (neat) 3061, 3026, 2947, 2867, 1688, 1597, 1495, 1445, 1199, 1159, 1135, 967, 881, 795, 750, 718, 701, 689, 653, $567 \mathrm{~cm}^{-1}$; HRMS (EI) calcd for $\mathrm{C}_{30} \mathrm{H}_{39} \mathrm{~N}_{2} \mathrm{OSiBr}(\mathrm{M})^{+}$664.2485, found 664.2452.

\section{2-[6-Bromo-1-(triisopropylsilyl)-1H-indol-3-yl]-2-} (dibenzylamino)-1-phenylethan-1-one 9e

Yield 73\% (97.0 mg); yellow oil; $R_{\mathrm{f}}=0.62$ (nhexane : ethyl acetate $=6: 1) ;{ }^{1} \mathrm{H}$ NMR $\left(400 \mathrm{MHz}, \mathrm{CDCl}_{3}\right) \delta 0.88-1.09(\mathrm{~m}, 18 \mathrm{H})$, 1.47-1.59 (m, 3H), 3.96 (dd, $J=13.7,34.8 \mathrm{~Hz}, 4 \mathrm{H}), 5.73(\mathrm{~s}, 1 \mathrm{H})$, 6.84-7.68 (m, 18H); ${ }^{13} \mathrm{C}$ NMR (100 MHz, $\left.\mathrm{CDCl}_{3}\right) \delta 3047,2948$, 2868, 1686, 1599, 1456, 1151, 1073, 967, 883, 814, 750, 695, 599; IR (neat) 3047, 2948, 2868, 1686, 1599, 1456, 1151, 1073, 967, 883, 814, 750, 695, $599 \mathrm{~cm}^{-1}$; HRMS (EI) calcd for $\mathrm{C}_{30} \mathrm{H}_{39} \mathrm{~N}_{2^{-}}$ $\operatorname{OSiBr}(\mathrm{M})^{+}$664.2485, found 664.2488. 
Synthesis of methyl 2-(dibenzylamino)-1-phenylbutan-1-one $10 \mathrm{~b}$ (general procedure for the reaction of the amino silyl enol ether with grignard reagents)

Under an argon atmosphere, to a solution of $\mathrm{N}$-bromosuccinimide (19.6 mg, $0.11 \mathrm{mmol})$ in EtCN $(1.0 \mathrm{~mL})$ were successively added a solution of amino silyl enol ether 5 (42.9 $\mathrm{mg}, 0.10$ $\mathrm{mmol})$ in $\mathrm{EtCN}(1.0 \mathrm{~mL}), \mathrm{BF}_{3} \cdot \mathrm{OEt}_{2}(0.025 \mathrm{~mL}, 0.20 \mathrm{mmol})$, and a solution of EtMgBr in $\mathrm{Et}_{2} \mathrm{O}(0.93 \mathrm{M}, 0.22 \mathrm{~mL}, 0.20 \mathrm{mmol})$ at room temperature. The reaction mixture was stirred for $30 \mathrm{~min}$. It was quenched with sat. aq. $\mathrm{NH}_{4} \mathrm{Cl}(10 \mathrm{~mL})$. The layers were separated, and the aqueous layer was extracted with ethyl acetate $(30 \mathrm{~mL} \times 3)$. The combined organic extracts were washed with brine, dried over anhydrous $\mathrm{Na}_{2} \mathrm{SO}_{4}$, and concentrated in vacuo. The crude product was purified by preparative TLC on silica gel (developed twice with $n$ hexane : AcOEt $=$ $15: 1)$ to give 2-(dibenzylamino)-1-phenylbutan-1-one 10b (74\%, $25.5 \mathrm{mg})$.

Yield 74\% (25.5 mg); colorless oil; $R_{\mathrm{f}}=0.63$ ( $n$ hexane : ethyl acetate $=6: 1) ;{ }^{1} \mathrm{H} \mathrm{NMR}\left(400 \mathrm{MHz}, \mathrm{CDCl}_{3}\right) \delta 0.93(\mathrm{t}, J=7.3 \mathrm{~Hz}$, $3 \mathrm{H}), 1.80-1.89$ (m, 1H), 1.93-2.02 (m, 1H), 3.69 (s, 4H), 4.14 (dd, $J=4.9,8.5 \mathrm{~Hz}, 1 \mathrm{H}), 7.20-7.34$ (m, 12H), 7.48-7.52 (m, 1H), 7.56$7.58(\mathrm{~m}, 2 \mathrm{H}) ;{ }^{13} \mathrm{C} \mathrm{NMR}\left(100 \mathrm{MHz}, \mathrm{CDCl}_{3}\right): \delta 11.5,17.8,54.3,62.6$, 127.0, 128.2, 128.2, 128.5, 129.1, 132.6, 137.7, 139.6, 201.4; IR (neat): 3062, 3026, 2978, 2936, 2835, 1684, 1494, 1448, 1378, 1225, 1143, 936, 749, 732, $695 \mathrm{~cm}^{-1}$; HRMS (EI) calcd for $\mathrm{C}_{18} \mathrm{H}_{20} \mathrm{NO}\left(\mathrm{M}-\mathrm{C}_{6} \mathrm{H}_{5}\right)^{+}$238.1590, found 238.1589.

\section{2-(Dibenzylamino)-1-phenylpropan-1-one 10a}

Yield 64\% (22.2 mg); colorless oil; $R_{\mathrm{f}}=0.63$ (nhexane : ethyl acetate $=6: 1) ;{ }^{1} \mathrm{H} \mathrm{NMR}\left(400 \mathrm{MHz}, \mathrm{CDCl}_{3}\right) \delta 1.34(\mathrm{~d}, J=6.6 \mathrm{~Hz}$, $3 \mathrm{H}), 3.54(\mathrm{~d}, J=13.5 \mathrm{~Hz}, 2 \mathrm{H}), 3.68$ (d, $J=13.5 \mathrm{~Hz}, 2 \mathrm{H}), 4.34$ (q, $J$ $=6.6 \mathrm{~Hz}, 1 \mathrm{H}), 7.15-7.34(\mathrm{~m}, 12 \mathrm{H}), 7.48-7.52(\mathrm{~m}, 1 \mathrm{H}), 7.58-7.61$ $(\mathrm{m}, 2 \mathrm{H}) ;{ }^{13} \mathrm{C} \mathrm{NMR}\left(100 \mathrm{MHz}, \mathrm{CDCl}_{3}\right) \delta 8.4,54.3,57.2,127.1$, 128.0, 128.2, 128.8, 129.2, 132.5, 136.9, 139.3, 202.0; IR (neat) 3062, 3026, 2978, 2936, 2835, 1684, 1494, 1448, 1378, 1225, 1143, 936, 749, 732, $695 \mathrm{~cm}^{-1}$; HRMS (EI) calcd for $\mathrm{C}_{17} \mathrm{H}_{18} \mathrm{NO}$ $\left(\mathrm{M}-\mathrm{C}_{6} \mathrm{H}_{5}\right)^{+}$224.1434, found 224.1438 .

\section{2-(Dibenzylamino)-1-phenylpentan-1-one 10c}

Yield $71 \%$ (25.4 mg); colorless oil; $R_{\mathrm{f}}=0.64$ (nhexane : ethyl acetate $=6: 1) ;{ }^{1} \mathrm{H}$ NMR $\left(400 \mathrm{MHz}, \mathrm{CDCl}_{3}\right) \delta 0.92(\mathrm{t}, J=7.3 \mathrm{~Hz}$, $3 \mathrm{H}), 1.29-1.39$ (m, 2H), 1.74-1.82 (m, 1H), 1.88-1.97 (m, 1H), $3.70(\mathrm{~s}, 4 \mathrm{H}), 4.24(\mathrm{dd}, J=5.5,8.7 \mathrm{~Hz}, 1 \mathrm{H}), 7.21-7.34(\mathrm{~m}, 12 \mathrm{H})$, 7.48-7.58 (m, 3H). ${ }^{13} \mathrm{C} \mathrm{NMR}\left(100 \mathrm{MHz}, \mathrm{CDCl}_{3}\right): \delta$ 14.2, 20.1, 27.0, 54.3, 60.5, 127.0, 128.2, 128.2, 128.5, 129.1, 132.6, 137.5, 139.7, 201.7. IR (neat): 3061, 3028, 2957, 2832, 1684, 1494, 1447, 1373, 1245, 1209, 1072, 947, 751, $695 \mathrm{~cm}^{-1}$. HRMS (EI): calcd for $\mathrm{C}_{19} \mathrm{H}_{22} \mathrm{NO}\left(\mathrm{M}-\mathrm{C}_{6} \mathrm{H}_{5}\right)^{+}$252.1747, found 252.1753.

\section{2-(Dibenzylamino)-3-methyl-1-phenylbutan-1-one 10d}

Yield $63 \%$ (22.5 mg); colorless oil; $R_{\mathrm{f}}=0.63$ (nhexane : ethyl acetate $=6: 1) ;{ }^{1} \mathrm{H} \mathrm{NMR}\left(400 \mathrm{MHz}, \mathrm{CDCl}_{3}\right) \delta 0.75(\mathrm{~d}, J=6.7 \mathrm{~Hz}$, $3 \mathrm{H}), 1.24(\mathrm{~d}, J=6.7 \mathrm{~Hz}, 3 \mathrm{H}), 2.35-2.46(\mathrm{~m}, 1 \mathrm{H}), 3.34(\mathrm{~d}, J=$ $14.7 \mathrm{~Hz}, 2 \mathrm{H}), 4.02$ (d, $J=10.4 \mathrm{~Hz}, 1 \mathrm{H}), 4.04$ (d, $J=14.7 \mathrm{~Hz}, 2 \mathrm{H})$, 7.21-7.29 (m, 10H), 7.37-7.40 (m, 2H), 7.54-7.57 (m, 1H), 7.65- $7.67(\mathrm{~m}, 2 \mathrm{H}) ;{ }^{13} \mathrm{C} \mathrm{NMR}\left(100 \mathrm{MHz}, \mathrm{CDCl}_{3}\right) \delta 20.3,20.4,27.8,54.3$, 65.4, 126.9, 128.1, 128.2 , 128.5, 128.6, 133.0, 139.6, 139.9, 203.1; IR (neat): 3059, 3032, 2979, 2950, 2840, 1668, 1494, 1444, 1218, 1093, 976, 839, 737, 730, $699 \mathrm{~cm}^{-1}$; HRMS (EI) calcd for $\mathrm{C}_{19} \mathrm{H}_{22} \mathrm{NO}\left(\mathrm{M}-\mathrm{C}_{6} \mathrm{H}_{5}\right)^{+}$252.1747, found 252.1741.

\section{2-Cyclopropyl-2-(dibenzylamino)-1-phenylethanone 10e}

Yield 35\% (12.4 mg); colorless oil; $R_{\mathrm{f}}=0.64$ (nhexane : ethyl acetate $=6: 1) ;{ }^{1} \mathrm{H}$ NMR $\left(400 \mathrm{MHz}, \mathrm{CDCl}_{3}\right) \delta-0.02-0.04(\mathrm{~m}$, $1 \mathrm{H}), 0.51-0.58(\mathrm{~m}, 2 \mathrm{H}), 0.75-0.84(\mathrm{~m}, 1 \mathrm{H}), 1.28-1.37(\mathrm{~m}, 1 \mathrm{H})$, 3.45 (d, $J=9.6 \mathrm{~Hz}, 1 \mathrm{H}), 3.79(\mathrm{~d}, J=13.8 \mathrm{~Hz}, 2 \mathrm{H}), 3.94(\mathrm{~d}, J=$ $13.8 \mathrm{~Hz}, 2 \mathrm{H}), 7.20-7.36(\mathrm{~m}, 12 \mathrm{H}), 7.50-7.61(\mathrm{~m}, 3 \mathrm{H}) ;{ }^{13} \mathrm{C}$ NMR $\left(100 \mathrm{MHz}, \mathrm{CDCl}_{3}\right) \delta 2.9,5.3,8.5,54.7,66.7,127.0,128.1,128.2$, 128.6, 129.0, 132.7, 137.5, 139.8, 201.7; IR (neat) 3081, 3027, 2927, 2840, 1681, 1597, 1495, 1450, 1220, 744, $696 \mathrm{~cm}^{-1}$; HRMS (EI) calcd for $\mathrm{C}_{19} \mathrm{H}_{20} \mathrm{NO}\left(\mathrm{M}-\mathrm{C}_{6} \mathrm{H}_{5}\right)^{+} 250.1590$, found 250.1591 .

\section{2-Cyclohexyl-2-(dibenzylamino)-1-phenylethanone $10 \mathrm{f}$}

Yield $60 \%$ (23.9 mg); colorless solid, $\mathrm{mp}=88-89{ }^{\circ} \mathrm{C} ; R_{\mathrm{f}}=0.65$ (nhexane : ethyl acetate $=6: 1) ;{ }^{1} \mathrm{H}$ NMR $\left(500 \mathrm{MHz}, \mathrm{CDCl}_{3}\right.$ ) $\delta$ 0.76-0.84 (m, 1H), 1.04-1.37 (m, 5H), 1.54-1.56 (m, 1H), 1.63$1.66(\mathrm{~m}, 1 \mathrm{H}), 1.79-1.83(\mathrm{~m}, 1 \mathrm{H}), 2.09-2.17$ (m, 1H), 2.42-2.45 $(\mathrm{m}, 1 \mathrm{H}), 3.36$ (d, $J=14.7 \mathrm{~Hz}, 2 \mathrm{H}), 4.05$ (d, $J=14.7 \mathrm{~Hz}, 2 \mathrm{H}), 4.16$ $(\mathrm{d}, J=10.4 \mathrm{~Hz}, 1 \mathrm{H}), 7.22-7.39(\mathrm{~m}, 12 \mathrm{H}), 7.52-7.55(\mathrm{~m}, 1 \mathrm{H}), 7.63-$ $7.65(\mathrm{~m}, 2 \mathrm{H}) ;{ }^{13} \mathrm{C} \mathrm{NMR}\left(126 \mathrm{MHz}, \mathrm{CDCl}_{3}\right) \delta 26.0,26.1,26.6,30.4$, 31.0, 37.4, 54.3, 64.2, 126.8, 128.1, 128.2, 128.5, 128.6, 133.0, 139.6, 139.9, 203.4; IR (neat) 3061, 3028, 2926, 2850, 1675, 1494, 1446, 1234, 1201, 907, 844, 735, $698 \mathrm{~cm}^{-1}$; HRMS (EI): calcd for $\mathrm{C}_{22} \mathrm{H}_{26} \mathrm{NO}\left(\mathrm{M}-\mathrm{C}_{6} \mathrm{H}_{5}\right)^{+}$292.2060, found 292.2056.

\section{2-(Dibenzylamino)-1,3-diphenylpropan-1-one $10 \mathrm{~g}$}

Yield $43 \%(17.4 \mathrm{mg})$; white solid $\mathrm{mp}=84-85{ }^{\circ} \mathrm{C} ; R_{\mathrm{f}}=0.61$ ( $n$ hexane : ethyl acetate $=6: 1$ ); ${ }^{1} \mathrm{H}$ NMR $\left(400 \mathrm{MHz}, \mathrm{CDCl}_{3}\right.$ ): $\delta 3.12(\mathrm{dd}, J=4.3,13.4 \mathrm{~Hz}, 1 \mathrm{H}), 3.36(\mathrm{dd}, J=9.2,13.4 \mathrm{~Hz}, 1 \mathrm{H})$, $3.71(\mathrm{~d}, J=13.4 \mathrm{~Hz}, 2 \mathrm{H}), 3.78(\mathrm{~d}, J=13.4 \mathrm{~Hz}, 2 \mathrm{H}), 4.54(\mathrm{dd}, J=$ 4.3, 9.2 Hz, 1H), 7.12-7.14 (m, 5H), 7.18-7.27 (m, 12H), 7.43$7.47(\mathrm{~m}, 3 \mathrm{H}) ;{ }^{13} \mathrm{C} \mathrm{NMR}\left(100 \mathrm{MHz}, \mathrm{CDCl}_{3}\right) \delta 30.2,54.3,62.9$, 126.0, 127.2, 128.1, 128.2, 128.3, 128.6, 129.2, 129.5, 132.6, 137.3, 139.1, 139.2, 199.7; IR (neat) 3060, 3025, 2931, 2815, 1688, 1600, 1494, 1455, 1233, 1116, 1072, 1027, 957, 750, $700 \mathrm{~cm}^{-1}$; HRMS (EI) calcd for $\mathrm{C}_{23} \mathrm{H}_{22} \mathrm{NO}\left(\mathrm{M}-\mathrm{C}_{6} \mathrm{H}_{5}\right)^{+} 300.1747$, found 300.1761 .

\section{2-(Dibenzylamino)-1-phenyl-2-p-tolylethanone 10i}

Yield 46\% (18.7 mg); yellow solid, $\mathrm{mp}=112-113{ }^{\circ} \mathrm{C} ; R_{\mathrm{f}}=0.62$ (nhexane : ethyl acetate $=6: 1) ;{ }^{1} \mathrm{H}$ NMR $\left(400 \mathrm{MHz}, \mathrm{CDCl}_{3}\right)$ $\delta 2.30(\mathrm{~s}, 3 \mathrm{H}), 3.75(\mathrm{~d}, J=13.7 \mathrm{~Hz}, 2 \mathrm{H}), 3.96(\mathrm{~d}, J=13.7 \mathrm{~Hz}, 2 \mathrm{H})$, 5.43 (s, 1H), 7.11-7.31 (m, 16H), 7.42-7.44 (m, 1H), 7.67-7.69 $(\mathrm{m}, 2 \mathrm{H}) ;{ }^{13} \mathrm{C}$ NMR $\left(100 \mathrm{MHz}, \mathrm{CDCl}_{3}\right) \delta 21.1,54.4,66.5,126.9$, 128.2, 128.3, 128.4, 128.9, 129.2, 129.9, 132.8, 133.3, 136.9, 137.5, 140.1, 201.7; IR (neat): 3059, 3026, 2922, 2844, 1689, 1595, 1494, 1447, 1226, 1138, 961, 804, 747, $700 \mathrm{~cm}^{-1}$; HRMS (EI): calcd for $\mathrm{C}_{23} \mathrm{H}_{22} \mathrm{NO}\left(\mathrm{M}-\mathrm{C}_{6} \mathrm{H}_{5}\right)^{+} 300.1747$, found 300.1744. 


\section{2-(Dibenzylamino)-1-phenyl-2-(thiophen-2-yl)ethanone $10 \mathrm{j}$}

Yield 64\% (25.5 mg); colorless solid, $\mathrm{mp}=108-109{ }^{\circ} \mathrm{C} ; R_{\mathrm{f}}=$ 0.64 (nhexane : ethyl acetate $=6: 1) ;{ }^{1} \mathrm{H}$ NMR $\left(400 \mathrm{MHz}, \mathrm{CDCl}_{3}\right)$ $\delta 3.67(\mathrm{~d}, J=13.7 \mathrm{~Hz}, 2 \mathrm{H}), 3.91(\mathrm{~d}, J=13.7 \mathrm{~Hz}, 2 \mathrm{H}), 5.69(\mathrm{~s}, 1 \mathrm{H})$, 6.90-6.92 (m, 1H), 6.96-6.99 (m, 1H), 7.23-7.37 (m, 13H), 7.48$7.52(\mathrm{~m}, 1 \mathrm{H}), 7.67-7.69(\mathrm{~m}, 2 \mathrm{H}) ;{ }^{13} \mathrm{C} \mathrm{NMR}\left(100 \mathrm{MHz}, \mathrm{CDCl}_{3}\right)$ $\delta 54.5,61.3,126.3,126.5,127.1,128.3,128.4,128.6,129.0,133.1$, 136.5, 137.5, 139.4, 198.9. IR (neat): 3061, 3027, 2928, 2840, 1685, 1595, 1494, 1447, 1214, 957, 750, $699 \mathrm{~cm}^{-1}$; HRMS (EI) calcd for $\mathrm{C}_{20} \mathrm{H}_{18} \mathrm{NOS}\left(\mathrm{M}-\mathrm{C}_{6} \mathrm{H}_{5}\right)^{+} 292.1154$, found 292.1140 .

\section{Conflicts of interest}

There are no conflicts to declare.

\section{Acknowledgements}

This work was supported by Grants-in-Aid for Scientific Research (B) and on Innovative Areas "Organic Synthesis Based on Reaction Integration. Development of New Methods and Creation of New Substances" from JSPS and MEXT.

\section{Notes and references}

1 For iminium ion cyclization, see: (a) E. J. Corey and R. D. Balanson, J. Am. Chem. Soc., 1974, 96, 6516-6517; (b) S. Yamada, M. Konda and T. Shioiri, Tetrahedron Lett., 1972, 13, 2215-2218. For $N$-acyl iminium ion cyclization, see; (c) J. Dijkink, H. E. Shoemeker and W. N. Speckamp, Tetrahedron Lett., 1975, 16, 4043-4046; (d) J. Dijkink and W. N. Speckamp, Tetrahedron Lett., 1975, 16, 4047-4050.

2 (a) H. H. Mooiweer, H. Hiemstra and W. N. Speckamp, Tetrahedron, 1989, 45, 4627-4636; (b) H. H. Mooiweer, E. C. Roots, H. Hiemstra and W. N. Speckamp, J. Org. Chem., 1992, 57, 6769-6778; (c) M. Okuda, H. Hioki, W. Miyagi and S. Ito, Tetrahedron Lett., 1993, 34, 61316134; (d) J. Yoshida, S. Suga and M. Okajima, Tetrahedron Lett., 2001, 42, 2173-2176; (e) J. Yoshida, S. Suga and M. Watanabe, J. Am. Chem. Soc., 2002, 124, 14824-14825; (f) P. Knochel, N. Millot, C. Piazza and S. Avolio, Synthesis, 2002, 941-944; $(g)$ L. W. Bieber and I. H. S. Estevam, Tetrahedron Lett., 2003, 44, 667-670; (h) M. Suginome, L. Uehlin and M. Murakami, Org. Lett., 2004, 6, 1167-1169; (i) T. Murai, Y. Mutoh, Y. Ohta and M. Murakami, J. Am. Chem. Soc., 2004, 126, 5968-5969; (j) M. Suginome, L. Uehlin and M. Murakami, J. Am. Chem. Soc., 2004, 126, 13196-13197.

3 For Petasis reactions including the synthesis of $\alpha$ monosubstituted amino acids, see: $(a)$ N. A. Petasis and I. Akritopoulou, Tetrahedron Lett., 1993, 34, 583-586; (b) N. A. Petasis and I. A. Zavialov, J. Am. Chem. Soc., 1997, 119, 445-446; (c) N. A. Petasis, A. Goodman and I. A. Zavialov, Tetrahedron, 1997, 53, 16463-16470; (d) N. A. Petasis and I. A. Zavialov, J. Am. Chem. Soc., 1998, 120, 11798-11799; (e) N. A. Petasis and S. Boral, Tetrahedron Lett., 2001, 42, 539-542; (f) T. Koolmeister,
M. Södelgren and M. Scobie, Tetrahedron Lett., 2002, 43, 5969-5970; $(g)$ H. Jourdan, G. Gouhier, L. V. Hijfte, P. Angibaud and S. R. Piettre, Tetrahedron Lett., 2005, 46, 8027-8031; (h) T. J. Southwood, M. C Curry and C. A. Hutton, Tetrahedron, 2006, 62, 236-242. For asymmetric Petasis reaction: ; $(i) \mathrm{S}$. Lou and S. E. Schaus, $J$. Am. Chem. Soc., 2008, 130, 6922-6923.

4 (a) Y. Niwa and M. Shimizu, J. Am. Chem. Soc., 2003, 125, 3720-3721; (b) M. Shimizu, H. Itou and M. Miura, J. Am. Chem. Soc., 2005, 127, 3296-3297; (c) T. Iwao and M. Shimizu, Heterocycles, 2009, 77, 767-772; (d) M. Shimizu, H. Itou, T. Iwao and Y. Umeda, Chem. Lett., 2009, 38, 732-733; (e) M. Shimizu, I. Hachiya and I. Mizota, Chem. Commun., 2009, 874-889; (f) S. Hata, H. Koyama and M. Shimizu, J. Org. Chem., 2011, 76, 9670-9677; (g) M. Shimizu, T. Kusunoki, M. Yoshida, K. Kondo and I. Mizota, Chem. Lett., 2011, 40, 351-353; (h) I. Mizota and M. Shimizu, Chem. Rec., 2016, 16, 688-702; (i) M. Shimizu, H. Imazato, I. Mizota and Y. Zhu, RSC Adv., 2019, 9, 1734117346; (j) M. Shimizu, M. Mushika, I. Mizota and Y. Zhu, RSC Adv., 2019, 9, 23400-23407; (k) M. Shimizu, Y. Furukawa, I. Mizota and Y. Zhu, New J. Chem., 2020, 44, 152-161; (l) M. Shimizu, T. Morimoto, Y. Yanagi, I. Mizota and Y. Zhu, RSC Adv., 2020, 10, 9955-9963.

5 We have already reported the formation of the iminium salt by ${ }^{1} \mathrm{H}$ and ${ }^{13} \mathrm{C}$ NMR spectra and a possible ionic mechanism by the ineffectiveness of radical scavengers. ${ }^{4 b}$

6 Effects of the silyl substituents on the reactivity of silyl enol ethers, see: (a) I. Kuwajima and E. Nakamura, Acc. Chem. Res., 1985, 18, 181-187; (b) M. B. Boxer and H. Yamamoto, J. Am. Chem. Soc., 2006, 128, 48-49; (c) M. Akakura, M. B. Boxer and H. Yamamoto, ARKIVOC, 2007, 337-347.

7 For the diastereoselecitivity of the Mannich reaction, see: $(a)$ A. Ting and S. E. Schaus, Eur. J. Org. Chem., 2007, 5797-5815; (b) J. M. M. Verkade, L. J. C. van Hemert, P. J. L. M. Quaedflieg and F. P. J. T. Rutjes, Chem. Soc. Rev., 2008, 37, 29-41; (c) Y. Gnas and F. Glorius, Synthesis, 2006, 1899-1930; (d) B. T. Hahn, R. Fröhlich, K. Harms and F. Glorius, Angew. Chem., Int. Ed., 2008, 47, 9985-9988.

8 The reaction of enolates and enols with DDQ was reported, see: (a) J. M. Williams, G. Marchesini, R. A. Reamer, U.-H. Dolling and E. J. J. Grabowski, J. Org. Chem., 1995, 60, 5337-5340; (b) A. Bhattacharya, L. M. DiMichele, U.-H. Dolling, A. W. Douglas and E. J. J. Grabowski, J. Am. Chem. Soc., 1988, 110, 3318-3319; (c) H.-D. Becker, J. Org. Chem., 1965, 30, 989-994.

9 (a) M. Bandini, A. Melloni, S. Tommasi and A. UmaniRonchi, Synlett, 2005, 1199; (b) J. C. Badenock and G. W. Gribble, Adv. Heterocycl. Chem., 2016, 120, 99-136, and references cited therein.

10 For determination of the relative stereochemistry, see: $(a)$ J. Delbos-Krampe, N. Risch and U. Florke, Z. Naturforsch., B: J. Chem. Sci., 2004, 59, 414-423; (b) M. Feroci, J. Lessard, M. Orsini and A. Inesi, Tetrahedron Lett., 2005, 46, 85178519. 